\title{
The Impact of Host Diet on Wolbachia Titer in Drosophila
}

\author{
Laura R. Serbus ${ }^{1,2 *}$, Pamela M. White ${ }^{3}$, Jessica Pintado Silva ${ }^{1,2}$, Amanda Rabe ${ }^{3}$, \\ Luis Teixeira ${ }^{4}$, Roger Albertson ${ }^{5}$, William Sullivan ${ }^{3}$ \\ 1 Department of Biological Sciences, Florida International University Modesto A. Maidique Campus, Miami, \\ Florida, United States of America, 2 Biomolecular Sciences Institute, Florida International University \\ Modesto A. Maidique Campus, Miami, Florida, United States of America, 3 Molecular, Cell, and \\ Developmental Biology, University of California Santa Cruz, Santa Cruz, California, United States of America, \\ 4 Instituto Gulbenkian de Ciência, Oeiras, Portugal, 5 Biology Department, Albion College, Albion, Michigan, \\ United States of America \\ *Iserbus@fiu.edu
}

\section{Abstract}

\section{G open access}

Citation: Serbus LR, White PM, Silva JP, Rabe A, Teixeira L, Albertson R, et al. (2015) The Impact of Host Diet on Wolbachia Titer in Drosophila. PLoS Pathog 11(3): e1004777. doi:10.1371/journal. ppat. 1004777

Editor: Elizabeth Ann McGraw, Monash University, AUSTRALIA

Received: September 20, 2014

Accepted: March 3, 2015

Published: March 31, 2015

Copyright: @ 2015 Serbus et al. This is an open access article distributed under the terms of the Creative Commons Attribution License, which permits unrestricted use, distribution, and reproduction in any medium, provided the original author and source are credited.

Data Availability Statement: All relevant data are within the paper and its Supporting Information files.

Funding: LRS received funding from Florida International University. AR received funding from the National Institutes of Health, Minority Access to Research Careers program, GMS-2T34GM007910. WS received funding from that National Science Foundation, MCB-1122252. The funders had no role in study design, data collection and analysis, decision to publish, or preparation of the manuscript.

Competing Interests: The authors have declared that no competing interests exist.

While a number of studies have identified host factors that influence endosymbiont titer, little is known concerning environmental influences on titer. Here we examined nutrient impact on maternally transmitted Wolbachia endosymbionts in Drosophila. We demonstrate that Drosophila reared on sucrose- and yeast-enriched diets exhibit increased and reduced Wolbachia titers in oogenesis, respectively. The yeast-induced Wolbachia depletion is mediated in large part by the somatic TOR and insulin signaling pathways. Disrupting TORC1 with the small molecule rapamycin dramatically increases oocyte Wolbachia titer, whereas hyper-activating somatic TORC1 suppresses oocyte titer. Furthermore, genetic ablation of insulin-producing cells located in the Drosophila brain abolished the yeast impact on oocyte titer. Exposure to yeast-enriched diets altered Wolbachia nucleoid morphology in oogenesis. Furthermore, dietary yeast increased somatic Wolbachia titer overall, though not in the central nervous system. These findings highlight the interactions between Wolbachia and germline cells as strongly nutrient-sensitive, and implicate conserved host signaling pathways by which nutrients influence Wolbachia titer.

\section{Author Summary}

Many invertebrate organisms carry bacterial endosymbionts within their cells. In many cases, this ensures host access to resources provided by the endosymbionts, and reciprocally, a rich source of host-supplied nutrients supports bacterial growth and reproduction. However if bacterial reproduction is uncontrolled, an over-abundance of bacteria will ultimately destroy the host cell. Here we explore the factors that regulate endosymbiont abundance in host cells. We focused on Wolbachia endosymbionts that are carried naturally in the germ cells of fruit flies. Specifically, we determined whether dietary nutrients affect the amount of Wolbachia bacteria carried by female flies. We found that yeast-enriched diets strongly depleted Wolbachia in fly ovarian cells. By contrast, sucrose-enriched diets 
doubled the amount of Wolbachia in ovarian cells. In addition, we found that this response to diet is mediated through highly conserved TORC1 and insulin signaling pathways in the fly. Recent studies have revealed that host diet dramatically influences the types and abundance of gut microbes. Our study informs how host diet affects endosymbiotic bacteria housed within specific types of host cells.

\section{Introduction}

Microbial endosymbionts have a profound impact on host metabolism and there are numerous examples in which microbes provide essential nutrients to the host [1-14]. In contrast, considerably less is known regarding how host metabolism and nutrition affect resident endosymbionts. To date, there is evidence that restricting the supply of host carbon, nitrogen and phosphorous significantly limits the number of Chlorella endosymbionts of green hydra and dinoflagellate endosymbionts of cnidarians [1]. Researchers have also observed that exposure to high levels of exogenous thiamine monophosphate suppresses the titer of Sodalis and Wigglesworthia endosymbionts in tsetse flies [15,16]. In this largely unexplored area, many outstanding questions remain: What are the host and endosymbiont metabolic and signaling pathways involved in nutrient sensing? To what extent do endosymbionts exhibit tissue-specific responses to nutrient availability? How are the rates of endosymbiont replication and cell death influenced by host metabolism and nutrients?

The symbiosis between Wolbachia and Drosophila is an excellent system to experimentally address these issues. Wolbachia are obligate intracellular endosymbionts carried by an estimated $40 \%$ of all insect species, including the established model organism Drosophila melanogaster [17-20]. Though Wolbachia endosymbionts are naturally carried within germline cells of both male and female insects, Wolbachia are ultimately removed from sperm prior to completion of spermatogenesis [17,18,21-25]. Thus, Wolbachia rely upon transmission through the maternal germline for their success. In addition to its functional importance in Wolbachia transmission, the well-characterized molecular and cell biology of Drosophila oogenesis has provided considerable contextual information and experimental tools that can be applied to studies of Wolbachia-host interactions [18,26-30].

The primary developmental units of the ovary that carry Wolbachia are referred to as egg chambers [27,28]. In each egg chamber, an outer layer of somatic follicle cells encapsulates an interconnected cyst of germline cells, comprised of 15 nurse cells and an oocyte. Wolbachia are initially loaded into these developing cysts during the first mitotic division from a Wolbachiainfected germline stem cell [18,31]. This germline Wolbachia population is amplified over time by binary fission and likely to some extent by exogenously invading Wolbachia [31-36]. Wolbachia persist in the germline throughout oogenesis, and a subset of the bacteria concentrate at the oocyte posterior pole during mid- to late oogenesis $[31,37,38]$. This ensures incorporation of Wolbachia into germline progenitor cells that form at the embryonic posterior pole, perpetuating the maternal germline transmission cycle [39]. Thus, maintenance of a sufficient Wolbachia titer in germline cells is important for success of the germline-based transmission strategy.

Here we examined how host diet affects Wolbachia titer in Drosophila melanogaster. The data demonstrate that yeast-enriched diets suppress Wolbachia titer and lead to altered nucleoid morphology during oogenesis. Genetic and chemical disruptions indicate that the somatic insulin and TORC1 pathways (Fig. 1) are required for yeast-based suppression of oocyte Wolbachia titer. The data also indicate that sucrose-enriched diets increased oocyte Wolbachia titer, with little impact on nucleoid morphology. Evidence indicates that yeast-enriched diets 


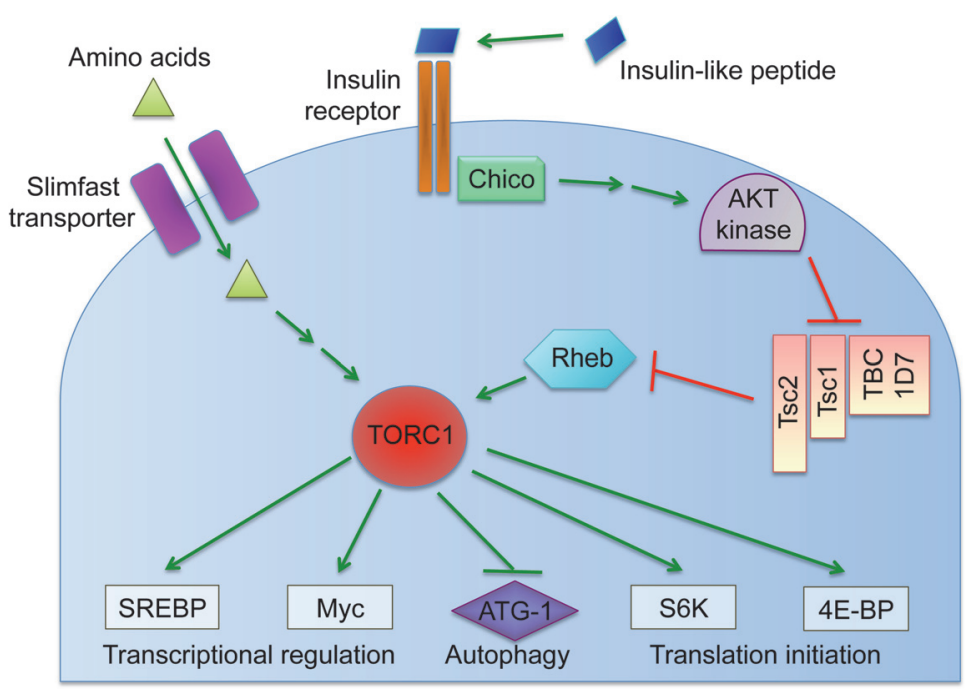

Fig 1. Overview of the nutrient-induced TORC1 signaling pathway.

doi:10.1371/journal.ppat.1004777.g001

substantially increase somatic Wolbachia titers, though this was not the case in the central nervous system (CNS). These studies demonstrate that Wolbachia, and likely other bacterial endosymbionts, exhibit distinct, tissue-specific responses to host nutrients that involve conserved signaling and metabolic pathways.

\section{Results}

\section{Exposing Drosophila to a yeast-enriched diet suppresses germline Wolbachia titer}

Nutrient availability strongly affects the life cycle of cultured bacteria, raising questions about how host nutrient conditions affect intracellular Wolbachia bacteria. As D. melanogaster in nature preferentially consume yeast [40-45], we tested the effect of dietary yeast on Wolbachia titer in vivo. Female flies were aged first for two days on standard food, then fed yeast paste for 3 days, and examined for Wolbachia titer in oogenesis. Ovarian tissues were stained with propidium iodide to label Wolbachia DNA, and the Wolbachia nucleoids imaged in oocytes of stage 10 egg chambers by confocal microscopy [38]. This analysis demonstrated that yeast paste-fed oocytes carried far less Wolbachia than control oocytes (Fig. 2A-B) (S1 Table). Wolbachia were further quantified within single oocyte focal planes to determine relative titer for each condition [32]. This revealed that Wolbachia titer in yeast paste-fed oocytes was at $27 \%$ of the control level. Oocytes treated with standard fly food exhibited an average of $229+/-21.1$ Wolbachia puncta $(\mathrm{n}=30)$, as compared to yeast paste-fed oocytes that carried $62.6+/-4.33$ Wolbachia $(\mathrm{n}=29)(\mathrm{p}<0.001)($ Fig. $2 \mathrm{C})$. This indicates that host exposure to yeast paste significantly reduces Wolbachia titer in oogenesis.

One possibility is that yeast paste diets reduce oocyte titer because other critical nutrients provided by standard fly food are unavailable. To address this issue, 2-day old Drosophila were fed with either standard food diluted $1 / 3$ with water, thereafter referred to as "control food", or fed with standard food diluted $1 / 3$ with yeast paste, thereafter referred to as "yeast-enriched food" (S1 Table). After 3 days of exposure to these conditions, titer was assessed in oogenesis. The yeast-enriched condition exhibited $55 \%$ of the control titer level, with controls displaying $124+/$ - 10.8 Wolbachia $(\mathrm{n}=58)$, compared to yeast-enriched oocytes carrying $68.7+/-5.12$ 

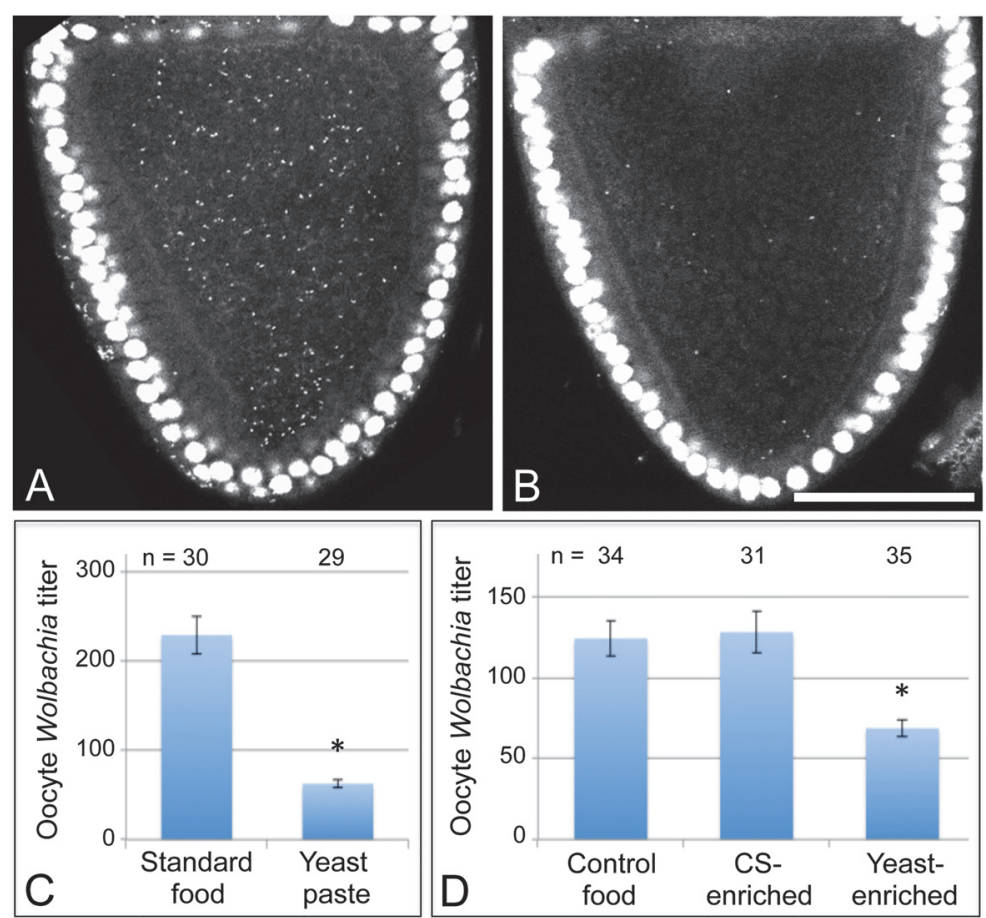

Fig 2. Host diet significantly impacts Wolbachia titer in Drosophila oogenesis. Stage $10 \mathrm{~A}$ oocytes are outlined in red. Propidium iodide indicates Drosophila nuclei as large circles and Wolbachia as small puncta. A) D. melanogaster oocyte exposed to standard fly food. B) D. melanogaster oocyte exposed to yeast paste. Graphs indicate the average number of Wolbachia nucleoids within single focal planes of stage 10A oocytes. C) Oocyte Wolbachia titer comparison between control food and yeast paste conditions. D) Wolbachia titer response in $D$. melanogaster to 1:3 dilutions of water, corn syrup (CS), or yeast paste into standard food. Scale bar: $50 \mu \mathrm{m}$.

doi:10.1371/journal.ppat.1004777.g002

Wolbachia $(\mathrm{n}=35)(\mathrm{p}=0.001)$ (Fig. 2D). To further assess whether this is due to differences in food hydration between control and yeast-enriched conditions, we also exposed flies to a $1 / 3$ dilution of corn syrup into standard fly food (S1 Table). Although corn syrup-enriched food is less hydrated than control food, it resulted in similar oocyte titer measurements as the control, with an average of $128+/-12.9$ Wolbachia visible per oocyte $(n=31)$ (Fig. 2D). These data together suggest that yeast-induced titer reduction is not due to depletion of specific nutrients or water available in standard food. Rather, the data indicate that dietary yeast is responsible for reducing Wolbachia titer carried by oocyte cells.

To determine whether dietary yeast can induce a similar oocyte titer response in wild insects as seen in laboratory fly stocks, Drosophila melanogaster and Drosophila simulans were collected from nature. These flies were exposed to yeast-enriched food and assessed for Wolbachia titer in oogenesis. We found that oocyte Wolbachia titer in the yeast-enriched condition was at $47 \%$ of the control level, with an average of $94.8+/-21.8$ Wolbachia detected in control oocytes $(\mathrm{n}=12)$, versus $44.6+/-6.52$ Wolbachia detected in the yeast-enriched condition $(\mathrm{n}=13)$

$(\mathrm{p}=0.029)$ (S1 Fig). Thus, yeast-enriched diets suppress oocyte Wolbachia titer in wild-caught Drosophila analogous to laboratory D. melanogaster strains.

To further investigate the basis for yeast-associated Wolbachia depletion in oocytes, Wolbachia titer was examined in the germline-derived nurse cells associated with the oocyte. It is currently unclear in Drosophila when or how frequently Wolbachia travel through the ring canals between the nurse cells and oocyte. Thus, it is possible that Wolbachia depletion in oocytes could be due to preferential retention in the nurse cells. To investigate this, we imaged 
Wolbachia in equivalent focal planes of nurse cells and oocytes within single egg chambers and analyzed their Wolbachia titer [32]. Overlaid images showing a planar reconstruction of egg chambers indicated fewer Wolbachia throughout the germline cells of yeast-exposed organisms (Fig. 3A-B). Quantitation of the yeast-enriched condition indicated that nurse cells carried $27 \%$ of the control titer level (Fig. 3C). Specifically, $52.6+/-4.93$ Wolbachia per nurse cell were detected in the control $(\mathrm{n}=20)$, in contrast to $14.4+/-1.65$ Wolbachia per nurse cell in the yeast-enriched condition $(\mathrm{n}=20)(\mathrm{p}<0.001)$ (Fig. 3C). Furthermore, oocyte titer in the yeastenriched condition was $14 \%$ of the control level, with $420+/-44.6$ Wolbachia detected in control oocytes $(\mathrm{n}=17)$, versus $59.0+/-11.1$ Wolbachia in oocytes from the yeast-enriched condition $(\mathrm{n}=20)(\mathrm{p}<0.001)$ (Fig. 3D). These data indicate that Wolbachia redistribution between germline cells is not responsible for the low oocyte titer observed in yeast-exposed organisms. Rather, yeast-enriched food induces similar Wolbachia depletion in nurse cells and oocytes.

\section{The nutrient-responsive kinase complex, TORC1, affects oocyte Wolbachia titer}

Cells coordinate intracellular events in response to exogenous nutrients using multiple signaling pathways that converge upon the Target of Rapamycin kinase complex 1 (TORC1) (Fig. 1) [46]. TORC1 can be activated by an amino-acid dependent signaling mechanism, or by insulin signaling (Fig. 1) [46-48]. To test whether TORC1 activity affects oocyte Wolbachia titer, flies were exposed to standard food containing the TORC1 inhibitor, rapamycin [49-52]. This experiment indicated that rapamycin treatment drove a 1.7 -fold increase in oocyte Wolbachia titer (Fig. 4A). The average titer from control oocytes, exposed to DMSO-containing standard food, was $207+/-22.1$ Wolbachia $(\mathrm{n}=28)$. By contrast, oocytes exposed to rapamycin-containing standard food had $357+/-31$ Wolbachia $(\mathrm{n}=30)(\mathrm{p}<0.01)($ Fig. 4A). Since rapamycin exposure leads to higher oocyte Wolbachia titer, this suggests that a normal consequence of TORC1 activity is suppression of oocyte Wolbachia titer.

If TORC1 function normally leads to decreased oocyte Wolbachia titer, then hyper-activation of TORC1 would be expected to drive a further reduction of oocyte titer. Branched chain amino acids (BCAAs) taken up through the Slimfast transporter can induce up-regulation of TORC1 (Fig. 1) [53-58]. Therefore, we fed flies a slurry of BCAAs diluted $1 / 3$ into standard food (S1 Table), and assessed Wolbachia titer in oogenesis. Wolbachia titer in the BCAA condition was reduced to $77 \%$ of the control (Fig. 4B). This was indicated by an average of $137+/-$ 9.71 Wolbachia in control oocytes $(\mathrm{n}=34)$ versus $105+/-8.48$ Wolbachia in oocytes from the BCAA condition $(\mathrm{n}=33)(\mathrm{p}=0.015)$ (Fig. 4B). The data suggest that TORC1 stimulation with BCAAs drives oocyte titer reduction, opposite the effects of the TORC1 inhibitor, Rapamycin.

To further investigate a possible role for TORC1, we genetically manipulated a key regulator of TORC1 activity. Tsc2, known as Gigas in Drosophila, is downstream of the insulin receptor (Fig. 1) [59-64]. If Tsc2 function is suppressed by any means, this allows TORC1 to become active (Fig. 1) [46,64-68]. Therefore, we tested the impact of Tsc2 on oocyte Wolbachia titer by expressing Tsc2 dsRNA under the control of germline- and soma-specific GAL4 drivers [6972]. This investigation revealed different oocyte Wolbachia titer responses to tissue-specific Tsc2 RNAi knockdowns. Our efforts to manipulate Tsc2 dosage in germline cells had no impact on oocyte titer (Fig. 4C). An average of $182+/-13.5$ Wolbachia were detected in control oocytes $(n=53)$, which was not significantly different from the $207+/-17.7$ Wolbachia detected in response to germline Tsc2 RNAi ( $\mathrm{n}=56)$ (Fig. 4C). By contrast, Tsc2 RNAi knockdowns in the somatic cells reduced oocyte Wolbachia titer to approximately $50 \%$ of the control level (Fig. 4D). Control oocytes exhibited an average of $402+/-43.4$ Wolbachia $(\mathrm{n}=24)$. However, oocytes somatic Tsc2 knockdown flies exhibited an average of $181+/-19.8$ oocyte 

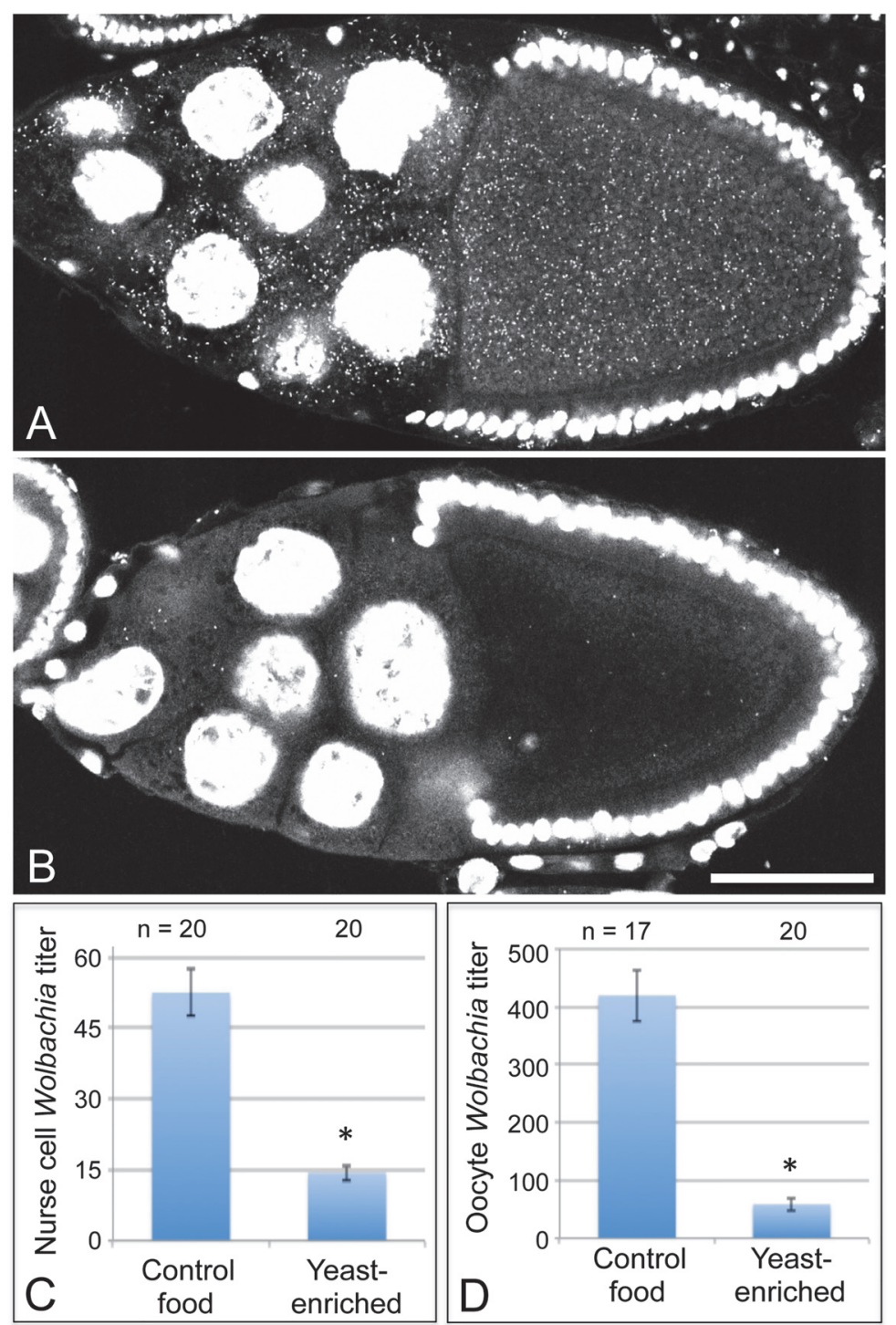

Fig 3. Dietary yeast affects Wolbachia titer in nurse cells as well as oocytes. Merged images show a full cross section from egg chambers raised on A) control food and B) yeast-enriched food. C-D) Average Wolbachia titer was determined for control vs. yeast-enriched conditions within a single egg chamber focal plane. C) Nurse cell titer values. D) Oocyte titer values from the same focal plane. Scale bar: $50 \mu \mathrm{m}$.

doi:10.1371/journal.ppat.1004777.g003

Wolbachia $(\mathrm{n}=21)(\mathrm{p}<0.001)$ (Fig. 4D). As such, these data implicate somatic Tsc2, and thus somatic TORC1 signaling, in regulation of oocyte Wolbachia titer.

\section{Yeast suppression of oocyte Wolbachia titer is mediated by insulin- TORC1 signaling}

A role for somatic TORC1 in regulating oocyte Wolbachia titer raised the question of whether dietary yeast stimulates TORC1. This could occur through either protein- or insulin-based mechanisms (Fig. 1). As yeast is major source of protein for D. melanogaster, perhaps its amino acid content stimulates TORC1 to ultimately suppress oocyte Wolbachia titer. To test this possibility, we exposed flies to food enriched in Bovine Serum Albumin, prepared specifically to match the protein content of yeast-enriched food (S1 Table). Oocyte Wolbachia titer was 

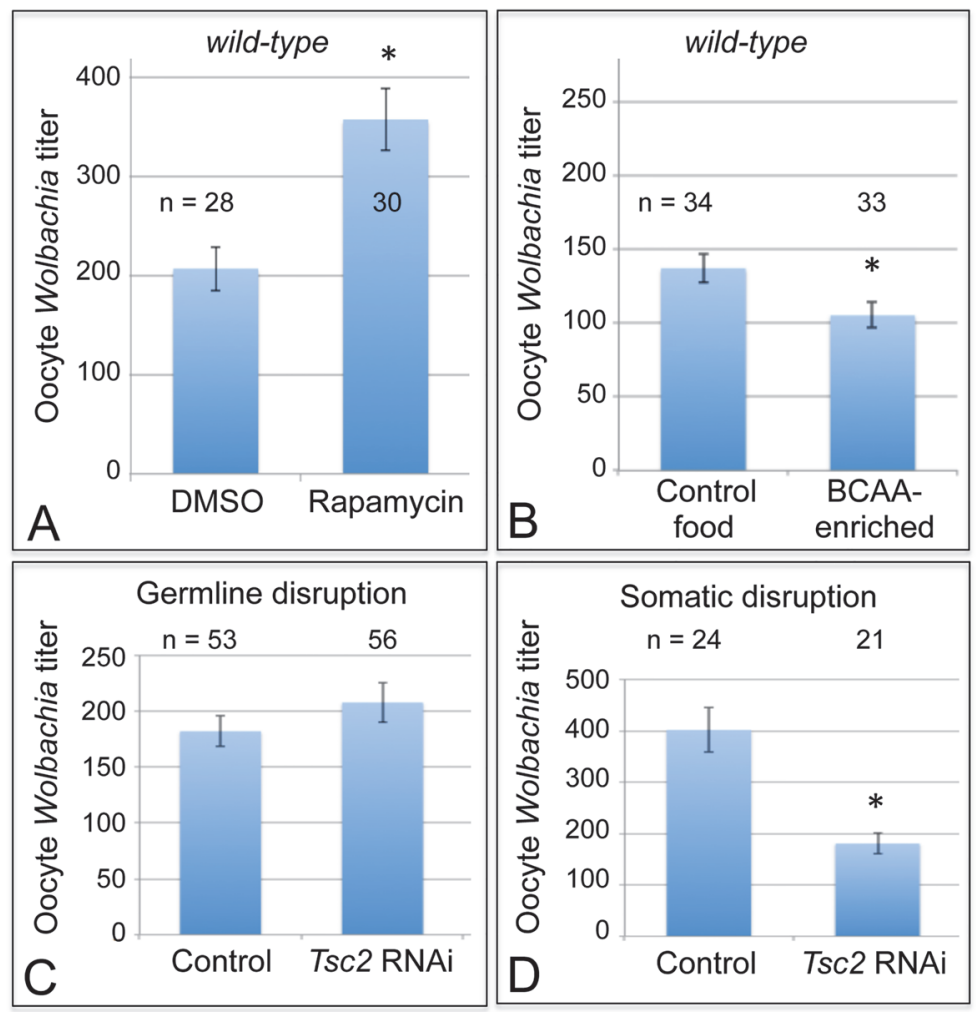

Fig 4. Somatic TORC1 activity affects Wolbachia titer in oogenesis. A) Average Wolbachia titer in oocytes treated with control DMSO or the mTORC1 inhibitor, Rapamycin. B) Titer was assessed in oocytes exposed to BCAA-enriched food. C-D) Wolbachia titer was also tested in flies carrying disruptions of the Tsc2 gene, expected to elevate TORC1 activity. C) Genotypes used for germline Tsc2 disruption: Control: \{nosGAL4\}/+; \{nos-GAL4\}/+. Tsc2 RNAi: \{nos-GAL4\}/+; \{nos-GAL4\}/\{UAS-Tsc2 dsRNA\}. D) Genotypes used for somatic Tsc2 disruption: Control: $\{d a-G A L 4\} /+$. Tsc2: $\{d a-G A L 4\} /\{U A S-T s c 2 d s R N A\}$. * indicates a significant change in titer.

doi:10.1371/journal.ppat.1004777.g004

similar for control and BSA-enriched conditions, however, with the control exhibiting $1260+/$ 102 Wolbachia $(\mathrm{n}=26)$, and the BSA-enriched condition exhibiting $1190+/-48.2$ Wolbachia $(\mathrm{n}=18)($ S2 Fig). This suggests that amino acid availability in the host diet has little impact on oocyte Wolbachia titer.

An alternate possibility is that yeast-enriched diets affect oocyte Wolbachia through insulin stimulation of TORC1. It was previously shown that dietary yeast stimulates insulin-producing cells (IPCs) the brain to release the insulin-like-peptides (Dilps) into the hemolymph [73,74].

To test whether yeast acts through somatic Dilp secretion to oocyte Wolbachia titer, we ablated the IPCs in the brain of fully mature Drosophila females. This is achieved using a dilp2: GeneSwitch-GAL4, UAS: Reaper system that specifically kills off the brain IPCs in response to a 2-week mifepristone treatment [74].

We first investigated whether mifepristone on its own modulates the yeast effect in wildtype flies. After completing a two-week exposure to either DMSO or mifepristone, flies were exposed to either control or yeast-enriched food for 3 days, and their oocyte titer levels were assessed. DMSO-treated flies exhibited substantial oocyte titer depletion in response to yeast-enriched food, down to $30 \%$ of the titer in the control condition (Fig. 5A). This was indicated by $785+/-64.8$ Wolbachia per oocyte in the DMSO-control food condition $(\mathrm{n}=24)$, in contrast to $191+/-26.9$ Wolbachia in the DMSO-yeast-enriched condition $(\mathrm{n}=25)(\mathrm{p}<.001)$ 

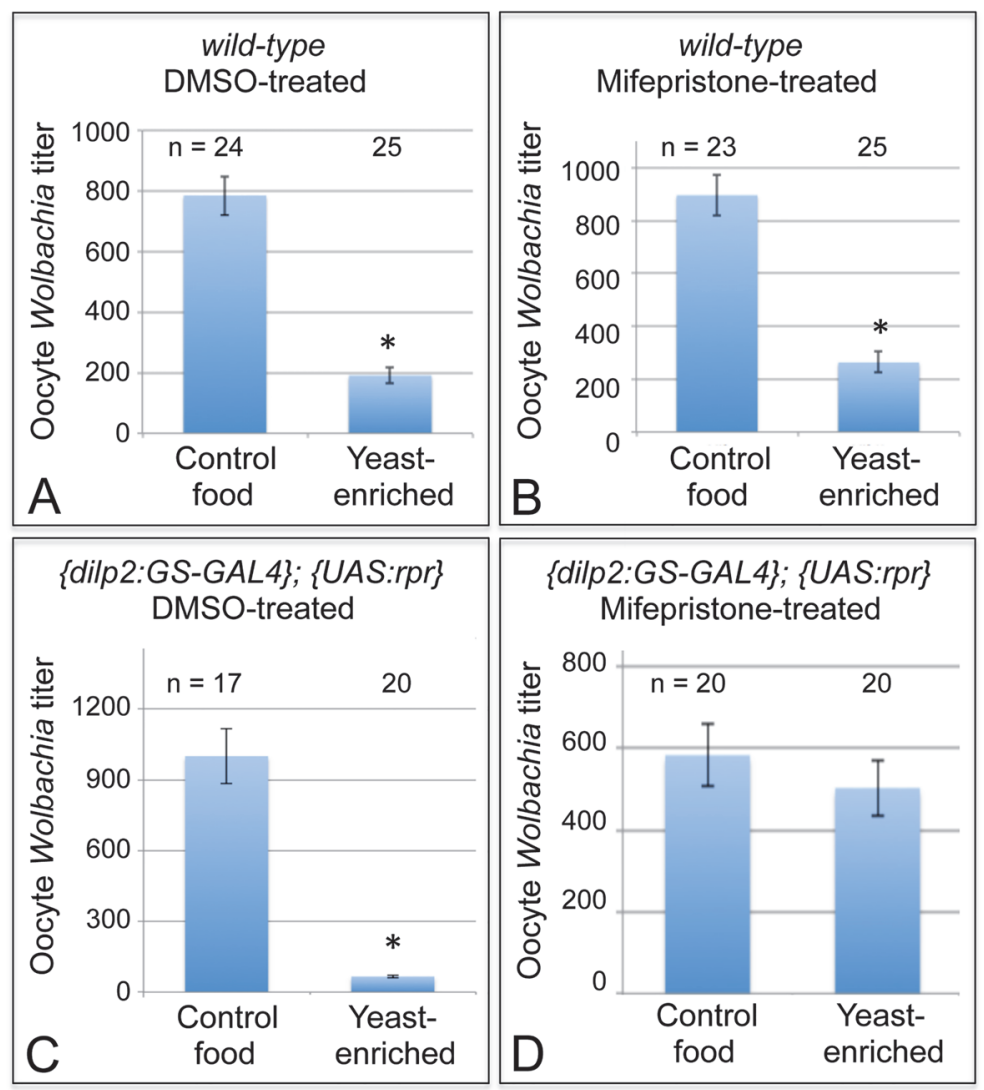

Fig 5. Nutrients affect germline Wolbachia titer through the somatic insulin pathway. Dietary impact on oocyte Wolbachia titer was tested in flies that either carried or lacked functional IPCs in the brain. Wild-type flies were A) treated with DMSO or B) induced with Mifepristone over a 14-day period as a control. \{dilp2: GS-GAL4\}; \{UAS-rpr\} flies were also C) treated with DMSO as a control, or D) induced with Mifepristone over a 14-day period to drive IPC lethality. * indicates significant changes in titer.

doi:10.1371/journal.ppat.1004777.g005

(Fig. 5A). Mifepristone-treated flies showed a similar titer reduction after exposure to yeast, exhibiting $21 \%$ of the titer seen in the control food condition (Fig. 5B). This was indicated by $896+/-77.2$ Wolbachia per oocyte in the mifepristone-control food condition $(\mathrm{n}=23)$, versus $264+/-39.5$ Wolbachia in the mifepristone-yeast-enriched condition $(\mathrm{n}=25)$ (Fig. 5B) $(\mathrm{p}<.001)$. Therefore, mifepristone alone has no effect on yeast-based suppression of oocyte Wolbachia titer.

Next, the exact same treatment regimens were performed on flies with the dilp2: GeneSwitch-GAL4, UAS: Reaper genotype. In this experiment, DMSO-treated flies, which retained functional IPCs, exhibited a severe oocyte Wolbachia depletion in response to yeast-enriched food, exhibiting only 7\% of the oocyte titer seen on DMSO-control food (Fig. 5C). This was indicated by the presence of $999+/-116$ Wolbachia per oocyte in the DMSO-control food condition $(\mathrm{n}=17)$, versus $66.5+/-6.61$ Wolbachia in the DMSO-yeast-enriched condition $(\mathrm{n}=20)$ $(\mathrm{p}<0.001)$ (Fig. 5C). In stark contrast, mifepristone-treated flies that had lost their IPCs exhibited no oocyte titer change after exposure to yeast (Fig. 5D). This was indicated by detection of $583+/-72.6$ Wolbachia per oocyte in the mifepristone-control food condition $(\mathrm{n}=20)$, versus $503+/-68.0$ Wolbachia in the mifepristone-yeast-enriched condition $(\mathrm{n}=20)$ (Fig. 5D). Since mifepristone in combination with the dilp2: Gene-Switch-GAL4, UAS: Reaper system 
specifically prevented yeast from affecting oocyte Wolbachia titer, this demonstrates that somatic IPCs mediate Wolbachia titer suppression by dietary yeast.

\section{Dietary sucrose elevates oocyte Wolbachia titer in an insulin-dependent manner}

To further investigate the sensitivity of oocyte Wolbachia titer to somatic insulin signaling, we also examined the effect of a sucrose-rich, high sugar diet. High sugar diets have been shown to induce insulin resistance in Drosophila [75,76]. This is may be due in part to increased expression of NLaz [75], which in mammals is known to suppress Akt function within the insulin signaling pathway (Fig. 1) [77-79]. To test the impact of sucrose-enriched diets on oocyte Wolbachia titer, 2-day old D. melanogaster were fed standard food diluted $1 / 3$ with saturated sucrose solution, hereafter referred to as "sucrose-enriched food" (S1 Table). After 3 days of exposure to this diet, Wolbachia titer was assessed in oogenesis. Oocytes from the sucrose-enriched condition exhibited a 2.4-fold increase in Wolbachia (Fig. 6A). Unlike oocytes raised on control food, which exhibited an average of $165+/-22.2$ Wolbachia $(\mathrm{n}=24)$, D. melanogaster oocytes exposed to sucrose-enriched food exhibited $392+/-25.3$ Wolbachia $(\mathrm{n}=26)$ $(\mathrm{p}<0.001)$ (Fig. 6A). These data indicate that a high sugar diet significantly elevates oocyte Wolbachia titer, possibly via an insulin-related mechanism.

A sucrose-based impact on oocyte Wolbachia titer is surprising, as corn syrup-enriched food did not induce a similar effect (Fig. 2D). Notably, sucrose is a disaccharide, composed of glucose and fructose, whereas corn syrup consists mainly of glucose. To elucidate the basis for sucrose-induced titer effects in oogenesis, food enriched for glucose and fructose were also tested. However, none of the monosaccharide-enriched conditions significantly affected oocyte Wolbachia titer (Fig. 6B). Control food yielded an average oocyte titer of $478+/-27.6$ Wolbachia per oocyte $(\mathrm{n}=71)$. Similarly, oocytes in the glucose-enriched condition displayed $520+/$ 31.1 bacteria $(\mathrm{n}=33)$, the fructose-enriched food condition resulted in $478+/-33.0$ Wolbachia $(\mathrm{n}=29)$, and a mixture of glucose + fructose yielded $499+/-28.0$ Wolbachia $(\mathrm{n}=32)$. By contrast, oocytes from the sucrose-enriched condition presented $883+/$ - 95.4 Wolbachia $(\mathrm{n}=22)$ $(\mathrm{p}<.001)$ (Fig. 6B). This confirms that disaccharide sucrose molecule specifically elicits Wolbachia titer increases in oogenesis.

To further test the possibility that insulin signaling mediates sucrose impact on ovarian Wolbachia titer, we coupled genetic disruptions of the insulin pathway with sucrose-enriched food. Chico is a Drosophila homolog of the Insulin Receptor Substrate that relays signals from the Insulin Receptor to AKT kinase, and thus ultimately TORC1 (Fig. 1) [80,81]. Germline and soma-specific GAL4 drivers were used to drive expression of chico dsRNA [69-72], and oocyte Wolbachia titer was assayed in control and sucrose-enriched conditions. This test did not indicate any effect of germline chico RNAi on sucrose-induced oocyte titer elevation, with sucroseenriched food corresponding to 2.4-fold higher oocyte titer than the control (Fig. 6C). Germline chico RNAi oocytes exhibited $125+/-10.6$ Wolbachia when exposed to regular food $(\mathrm{n}=26)$ as compared to $299+/-27.2$ Wolbachia in response to sucrose-enriched food $(\mathrm{n}=19)$ $(\mathrm{p}<0.001)$ (Fig. 6C). By contrast, somatic chico RNAi eliminated sucrose-induced titer effects in oogenesis (Fig. 6D). Oocytes from somatic chico RNAi flies exhibited $180+/-12.9$ Wolbachia in the control condition $(\mathrm{n}=25)$, as compared to $169+/-12.5$ Wolbachia per oocyte in the sucrose-enriched condition $(\mathrm{n}=25)$ (Fig. 6D). Analysis of sibling controls further indicated that the genetic background for the somatic chico RNAi experiment was not responsible for differential oocyte titer responses to sucrose (Fig. 6E). In flies carrying the somatic da-GAL4 driver used for this experiment, the sucrose-enriched condition continued to exhibit 2-fold more Wolbachia than the control food condition. An average of $124+/-11.1$ Wolbachia were 

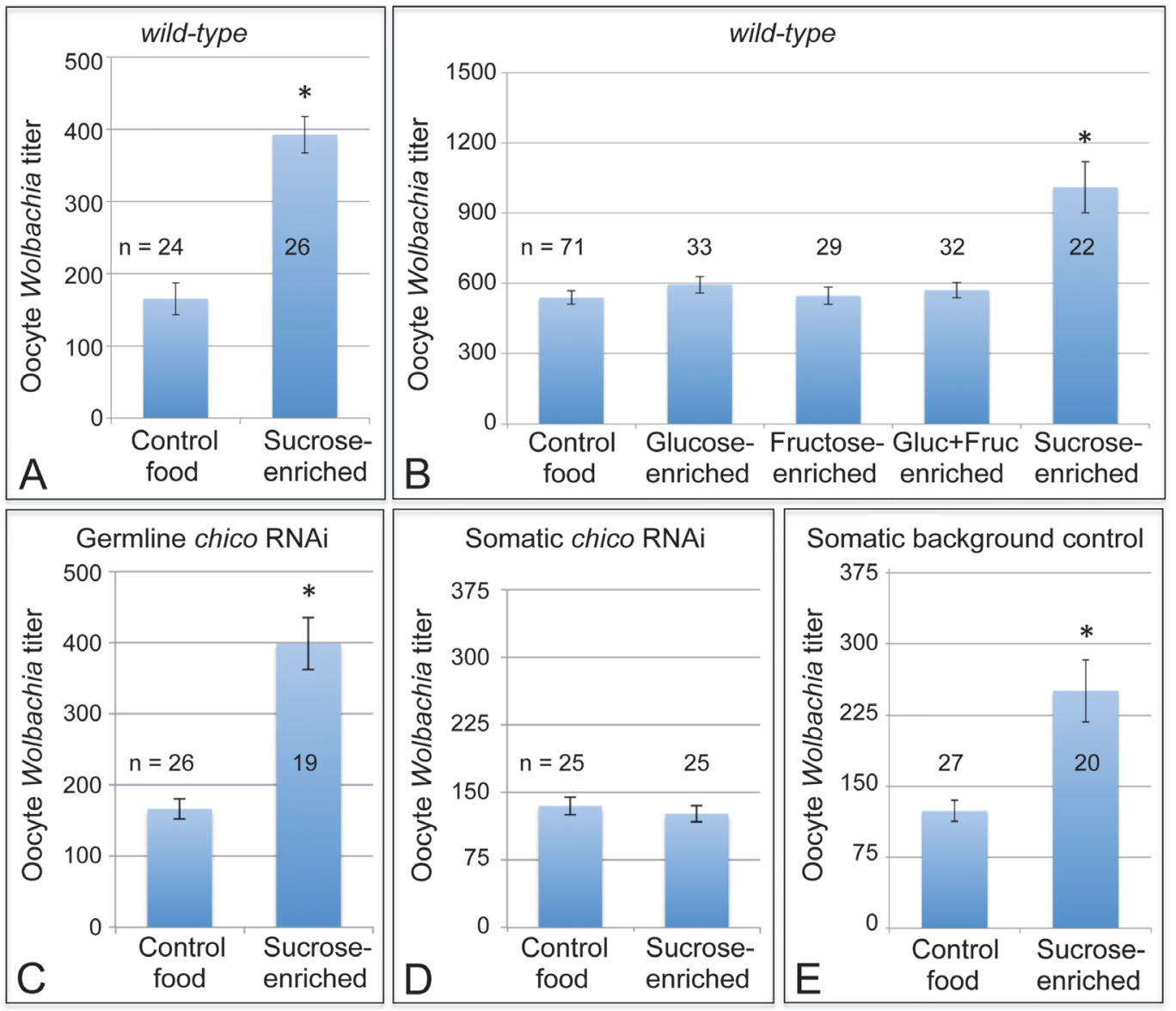

Fig 6. Sucrose-enriched food elevates oocyte Wolbachia titer in a chico-dependent manner. Wolbachia were quantified within single focal planes of oocytes exposed to control food or sucrose-enriched food. The average titer detected per nutrient condition is shown. A) Impact of sucrose on oocyte Wolbachia titer in wild-type D. melanogaster. B) Comparison of oocyte Wolbachia titers between control food and other foods enriched in glucose, fructose, a mixture of glucose and fructose, or sucrose. C-E) Sucrose impact on oocyte Wolbachia titer in flies that carry tissue-specific chico RNAi disruptions. Genotypes used: C) $\{$ nos-GAL4\}/+; \{nos-GAL4\}/\{UAS-chico dsRNA\}. D) $\{$ da-GAL4\}/\{UAS-chico dsRNA\}. E) $\{d a-G A L 4\} /+$.

doi:10.1371/journal.ppat.1004777.g006

detected in control oocytes $(\mathrm{n}=27)$ as compared to $251+/-32.8$ Wolbachia detected in oocytes from the sucrose-enriched condition $(\mathrm{n}=20)(\mathrm{p}<.001)$ (Fig. 6E). Though the complete mechanistic implications of somatic chico disruption remain unclear, these data demonstrate that sucrose acts through somatic insulin signaling to elevate oocyte Wolbachia titer.

\section{Oocyte Wolbachia titer responses are independent of ovary productivity}

These data raise the fundamental question of why diet-modulated insulin signaling affects Wolbachia titer so strongly in germline cells. One possibility is that these titer responses are an indirect result of nutrient-induced changes in ovary size and productivity [76]. Yeast-rich diets and insulin signaling are known to drive formation of larger, more productive ovaries [60,76,80,82-91], while high-sucrose diets have the opposite effect [76-79]. To test the contribution of ovary size and productivity variables on oocyte Wolbachia titer, we manipulated ovary productivity by controlling female mating. Mating stimulates ovary development, resulting in a moderately sized, productive ovary. By contrast, virgin females exhibit very large ovaries, filled mainly by mature eggs [92-96]. Oocytes from mated versus virgin females revealed similar oocyte Wolbachia titers, however (S3 Fig). The mated condition displayed 449 +/- 27.5 
Wolbachia per oocyte $(\mathrm{n}=26)$, while the virgin female condition that carried $470+/-40.6$ Wolbachia per oocyte $(\mathrm{n}=24)(\mathrm{S} 3 \mathrm{Fig})$. These data suggest that ovary size and productivity do not serve as the primary determinants of oocyte Wolbachia titer.

\section{Wolbachia nucleoid morphology responds to dietary yeast}

To further investigate the effects of host diet on Wolbachia, we examined Wolbachia nucleoid morphology. Other studies indicate that nucleoid morphology can serve as a proxy indicator of replication-associated changes in cell shape, or stress-induced DNA compaction [97-99]. Multiple, zoomed-in images of Wolbachia stained with propidium iodide were projected as a single image, and nucleoid shape was measured. The images indicated that Wolbachia nucleoid shape differs between nutrient conditions ( $\mathbf{S 4}$ Fig). To specifically analyze changes in nucleoid length, 120 nucleoids were selected at random from each treatment condition and their lengths were compared. This analysis indicated that $50 \%$ of nucleoids in the control condition exceeded $2 \mu \mathrm{m}$ in length (S4 Fig). The sucrose-enriched condition was similar, with $53 \%$ of nucleoids exceeding $2 \mu \mathrm{m}$. In the yeast-enriched condition, however, only $37 \%$ of nucleoids exceeded this measure $(\mathrm{p}<.05)$. Thus, yeast-enriched food significantly shortened Wolbachia nucleoids. We further determined an elongation index (EI), representing bacterial length divided by width, for the same 120 nucleoids per treatment condition as above. This analysis indicated that $50 \%$ of nucleoids measured in the control condition had an EI greater than 2. In the sucrose-enriched condition, only $33 \%$ of nucleoids showed an EI greater than $2(\mathrm{p}<.05)$. In the yeast-enriched condition, even fewer nucleoids showed this degree of elongation, with only $22 \%$ of nucleoids exceeding this EI ( $\mathrm{p}<.001)$ (S4 Fig). These data indicate that dietary conditions, and especially exposure to yeast-enriched food, alter Wolbachia nucleoid morphology in oogenesis. This is consistent with a bacterial physiological response to host diet.

\section{Wolbachia titers are regulated in a tissue-specific manner}

The striking impact of dietary nutrients on oocyte Wolbachia titer raises the question of whether Wolbachia titer in other tissues is responsive to nutrient conditions. Wolbachia are present in insect somatic cells, and the Drosophila brain is particularly amenable to assessment of somatic Wolbachia titer $[100,101]$. To take advantage of this, we imaged Wolbachia in the central brain of D. melanogaster exposed to different nutrient conditions. This analysis revealed that D. melanogaster on control food already carry very low Wolbachia titer in the central brain (Fig. 7A, $A^{\prime}, n=3$ ), and flies fed with either yeast-enriched or sucrose-enriched food were indistinguishable in appearance from the control (Fig. 7B, B', n=3) (Fig. 7C, C', n= 3). Thus, Wolbachia titer in D. melanogaster brain does not appear to be affected by the dietary conditions used in this study. An alternative possibility, however, is that the overall low Wolbachia titer detected under these conditions hampered our ability to assay nutrient-induced changes in titer.

To pursue this further, the impact of nutrient-altered food was tested in the closely related D. simulans species, known for carrying high Wolbachia titer in its brain cells [101]. Flies exposed to control food exhibited a high titer of Wolbachia in the central brain overall (Fig. 7D, $\left.\mathrm{D}^{\prime}, \mathrm{n}=7\right)$. Similarly high Wolbachia titer was detected in the brain after exposure to yeast-and sucrose-enriched food (Fig. 7E, E', n= 5) (Fig. 7F, F', n= 4). Further quantification of Wolbachia infection frequency did not reveal any differences between nutrient conditions (Fig. 7G). In control food, yeast-enriched, and sucrose-enriched conditions, 55-56\% of brain cells exhibited Wolbachia infection ( $\mathrm{n}=1171,767$, and 665 cells, respectively). No differences were seen in formation of large Wolbachia aggregates either (Fig. 7H). Brain samples reared on control food, yeast-enriched, and sucrose-enriched conditions all exhibited between 16-19 large 


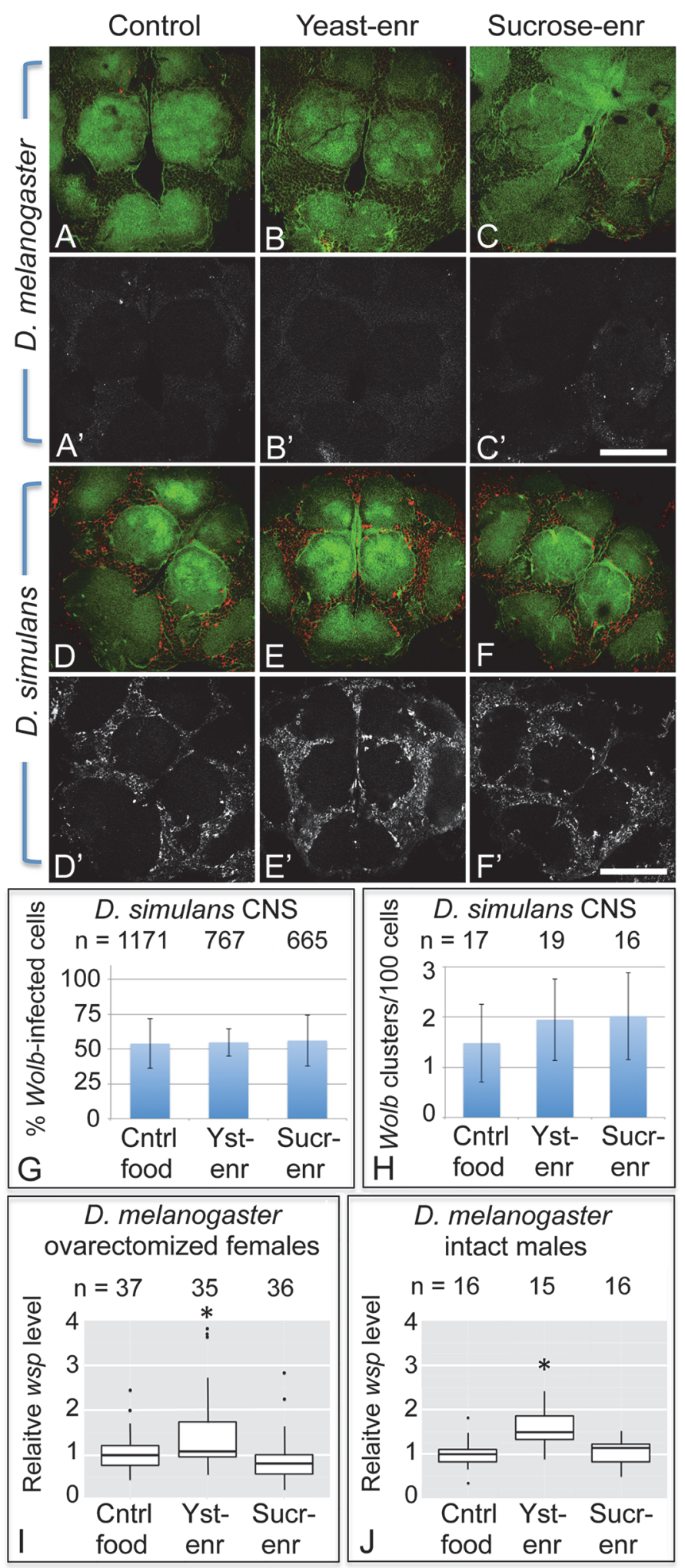

Fig 7. Host diet has tissue-specific effects on somatic Wolbachia titer. A-F') Wolbachia in the central brain of female flies. Columns from left to right: Control food, Yeast-enriched, Sucrose-enriched. In merged images, red shows Anti-Wsp to indicate Wolbachia, and green shows phalloidin to indicate actin. Grayscale images show only Anti-Wsp. A-C, A'-C') D. melanogaster brains. Little Wsp signal is detected under each 
feeding condition. D-F, D'-F') Brains from $D$. simulans. These show similarly high Wsp immunoreactivity under all feeding conditions. G) Percentage of Wolbachia-infected $D$. simulans brain cells. H) Frequency of large Wolbachia clusters per $100 \mathrm{D}$. simulans brain cells. I-J) qPCR analysis of relative Wolbachia levels from flies exposed to nutrient-altered diets. The Y-axis shows relative quantitation of genomic wsp. Flies used: I) ovarectomized $D$. melanogaster females. J) intact $D$. melanogaster males. Values are normalized to the control flies in each panel. * indicates a significant change in titer. Scale bars: $150 \mu \mathrm{m}$.

doi:10.1371/journal.ppat.1004777.g007

bacterial clusters per hundred cells. This indicates that Wolbachia titer in the D. simulans brain is unresponsive to the nutrient-altered conditions used in this study.

To address the possibility that $D$. simulans tissues are generally unresponsive to nutrients, we also assessed $D$. simulans oocyte titer in response to nutrient-altered food. In contrast to the brain, $D$. simulans oocytes exhibited a clear nutrient-dependent Wolbachia titer response (S5 Fig). Control oocyte images carried $293+/-49.9$ Wolbachia $(\mathrm{n}=10)$. By contrast, oocyte titer from the yeast-enriched condition was at $40 \%$ of the control level, with an average of $116+/$ 20.1 bacteria detected per oocyte $(\mathrm{n}=10)(\mathrm{p}=0.004)$. Furthermore, the sucrose-enriched condition exhibited 2.3-fold higher titer than the control, with $662+/-73.6$ Wolbachia detected per oocyte $(\mathrm{n}=10)(\mathrm{p}=0.001)$ (S5 Fig). Thus, D. simulans Wolbachia titers are capable of responding similarly to nutrient conditions as D. melanogaster.

To further probe the impact of host diet on somatic Wolbachia titer, we analyzed relative amounts of Wolbachia versus host DNA in ovarectomized female flies. In this analysis, females were exposed to nutrient-altered diets, dissected to remove ovarian tissues, and analyzed by qPCR. The results indicate the relative level of Wolbachia per host genome copy number. This analysis indicated that yeast-enriched dietary conditions led to higher levels of Wolbachia than the control food condition (Fig. 7I). Control samples exhibited a mean relative level of Wolbachia of $0.989(\mathrm{n}=37)$, whereas the yeast-enriched condition displayed a mean relative level of Wolbachia of $1.28(\mathrm{n}=35)(\mathrm{p}<0.05)$. Females exposed to sucrose-enriched diets were not significantly different from the control, however, exhibiting a mean Wolbachia relative level of $0.792(\mathrm{n}=36)$ (Fig. 7I). This titer response profile differs from analyses of Wolbachia titer in the ovary as well as the brain. This suggests that host diet affects Wolbachia titers in a tissuespecific manner.

As host nutrition has a different impact on ovarian versus somatic Wolbachia titers, this raises the question of what would happen in organism lacking ovarian tissue altogether. To address this issue, qPCR analysis was performed on intact male flies. This indicated that bodywide Wolbachia titer also increases in response to yeast-enriched food, although not sucroseenriched food (Fig. 7J). The control food condition carried a mean Wolbachia relative level of 1 $(\mathrm{n}=16)$, in contrast to the yeast-enriched condition, which displayed a mean Wolbachia relative level of $1.545(\mathrm{n}=15)(\mathrm{p}<0.05)$. Sucrose-enriched diets corresponded to a mean Wolbachia relative level of $1.027(\mathrm{n}=16)$. This analysis confirms that the profile of bodywide titer responses in males is equivalent to ovarectomized females. This suggests that somatic Wolbachia titers overall respond to host dietary conditions in a consistent manner.

\section{Discussion}

The major finding of this study is that dietary intake by Drosophila strongly influences Wolbachia titer in the host female germline: a high yeast diet decreases Wolbachia oocyte titer and a

high sucrose diet increases Wolbachia oocyte titer. This finding adds to a small but growing literature on the impact of host diet on endosymbionts $[1,15,16]$. Prior studies of Wolbachia suggest that this endosymbiont relies heavily upon host provisioning of amino acids and carbohydrates [102-104]. A very recent study analyzing the Drosophila midgut and ovary surprisingly indicated that neither dietary yeast nor sucrose had any affect on the Wolbachia:host 
genomic ratio in those tissues [105]. The image-based analyses of this study demonstrate that yeast and sucrose affect germline Wolbachia titer at the cellular level, however. It is unclear why Wolbachia titer in the oogenesis should be particularly sensitive to diet and whether this is an adaptive response to changes in the host metabolic environment. The evolutionary success of Wolbachia depends on its ability to localize at the posterior pole of the oocyte, the site of germline formation. Significantly, we find that Wolbachia localize to the posterior pole regardless of whether the host is exposed to the low titer, yeast-enriched diet, or the high titer, sucrose-enriched diet. This suggests the previously described microtubule and motor protein based mechanisms driving posterior localization of Wolbachia [38] are robust, even in the face of dramatic titer changes caused by nutrient-altered diets.

Insight into the mechanism of yeast-induced titer suppression comes from our functional studies demonstrating that this response is mediated through TORC1. Genetic up-regulation of TORC1 suppresses oocyte Wolbachia titer, whereas drug-based inhibition of TORC1 increases titer. This finding creates the basis for a sensible functional connection between intracellular Wolbachia and host diet, as both amino acids and insulin signaling are known to drive TORC1 activity [46]. Our finding that BSA-enriched food had no effect on oocyte Wolbachia titer argues that yeast protein content is not the major determinant of germline titer suppression, and alternatively suggests a role for insulin signaling. Prior work has shown that yeastrich diets trigger insulin signaling in Drosophila, and that Wolbachia interact with host insulin signaling processes $[89,106]$. Our finding, that loss of somatic IPCs eliminates yeast impact on oocyte Wolbachia titer, confirms that insulin signaling facilitates the titer-suppressing effects of yeast. Furthermore, disrupting the somatic insulin receptor substrate, Chico, suppressed the impact of dietary sucrose on oocyte Wolbachia titer. This suggests that both dietary yeast and sucrose affect germline Wolbachia titer via antagonistic impacts on somatic insulin signaling (Fig. 8).

In considering the mechanism of insulin-based impact on germline Wolbachia titer, one possibility is that changes in ovary productivity are responsible. Diet-modulated insulin signaling affects the relative rates of germline stem cell division, germline cell survival and egg chamber development $[60,76,80,82-91]$. If Wolbachia are unresponsive to nutrient-induced adjustments in germline cell growth and development, significant titer changes in oogenesis would be expected. However, oocyte Wolbachia titers were very similar in mated and virgin females, despite the different rates of germline stem division expected for each type of flies $[76,83,86,88,90-96]$. Another possibility is that yeast-induced insulin signaling affects Wolbachia physiology in oogenesis. The "rounded" Wolbachia nucleoids visible in the yeast-enriched condition could indicate substantially slowed bacterial growth or a bacterial stress response, for example [97-99]. Insulin signaling has been shown to induce changes in cytoskeleton organization, proteasome activity and chaperonin activity [107-111], any of which could affect Wolbachia physiology. It is also possible that dietary yeast in particular carries one or more bioreactive agents that are toxic to germline Wolbachia (Fig. 8).

The impact of somatic insulin signaling on germline Wolbachia titer also raises the question of whether somatic Wolbachia titers are similarly affected by host nutrient conditions. Our initial findings that Wolbachia titers in the Drosophila brain are non-responsive to host diet suggested that nutrient-associated titer changes are restricted to the ovary. Analysis of sucrose-fed, ovarectomized females is further consistent with that interpretation. However, analysis of ovarectomized females also indicated that dietary yeast triggers somatic titer changes opposite of oogenesis. It is possible that this occurs by physical relocation of Wolbachia within the body, with dietary yeast driving Wolbachia egress from ovarian cells, followed by invasion of somatic target tissues. Alternatively, host dietary conditions may drive tissue-specific differences in the Wolbachia life cycle. Perhaps yeast-enriched diets favor Wolbachia replication and survival in 


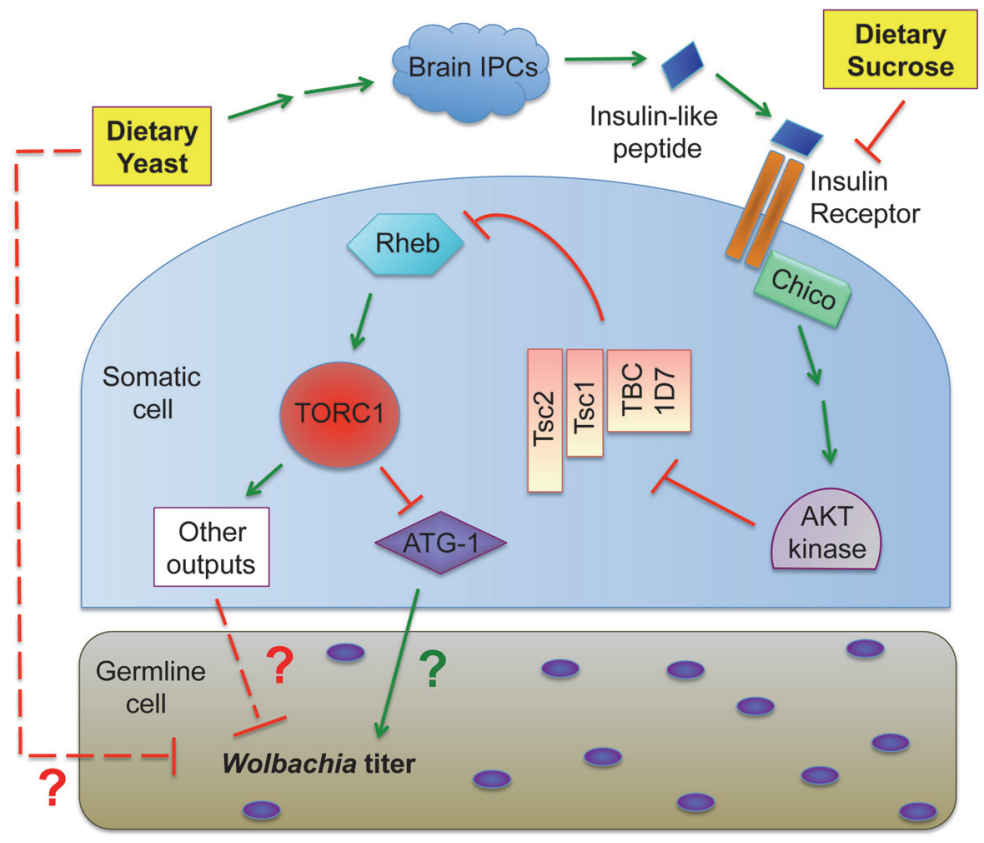

Fig 8. Model for the impact of host diet on germline Wolbachia titer.

doi:10.1371/journal.ppat.1004777.g008

specific somatic tissues while disfavoring the same in oogenesis. Support for this hypothesis comes from our finding that yeast-enriched food induces the same bodywide titer changes in male flies as seen in ovarectomized females. This demonstrates that ovarian Wolbachia titer responses are distinct from that of other tissues.

The pathways downstream and upstream of TORC1 that mediate yeast-based suppression of Wolbachia germline titer are yet to be determined. An obvious possibility is the role of TORC1 in suppressing autophagy (Fig. 8). There are numerous examples in which autophagy either enhances or suppresses intracellular bacteria titer [112]. Since TORC1 disruptions increase Wolbachia titer in oogenesis, it is possible that Wolbachia interact positively with autophagy, consistent with other endosymbionts [113] [114]. As insulin signaling is expected to down-regulate autophagy (Fig. 1), the low Wolbachia titers seen in yeast-fed oocytes are further consistent with this possibility. However, the finding that dietary yeast also increases somatic Wolbachia titers implies that somatic autophagy is normally bactericidal in that context, consistent with another recent report [115]. These conflicting results may indicate that tissue-specific differences in autophagy regulation contribute to Wolbachia titer control, or that other mechanisms downstream or independent from autophagy are responsible (Fig. 8). Perhaps responses from one or more other TORC1 effectors further contribute to Wolbachia titer regulation (Fig. 1).

Wolbachia have been shown to suppress replication of RNA viruses in insects, including the human pathogens, Dengue Fever Virus and Chikungunya Virus [116-118]. This finding, together with the fact that Wolbachia-induced Cytoplasmic Incompatibility rapid spreads Wolbachia through insect populations [25,119], has led to a novel strategy of combating these diseases by releasing Wolbachia-infected insect carriers of these viruses into afflicted regions $[120,121]$. Although the mechanism of Wolbachia-induced viral suppression is unknown, several studies demonstrate that the higher the Wolbachia titer, the greater the viral suppression [122-126]. Our finding that host diet dramatically affects tissue-specific Wolbachia titers suggests that the natural diets of the released insects should be taken into account when evaluating 
the potential effectiveness of a Wolbachia-based viral suppression field study. Finally it will be of interest to determine whether diet has a similar effect on Wolbachia titer in disease-associated filiarial nematodes.

\section{Materials and Methods}

\section{Fly strains}

Natural D. melanogaster and D. simulans flies were harvested daily from collection buckets distributed in the Santa Cruz, CA area. As the female flies of these species are morphologically indistinguishable, but both species were well-represented in the area, this wild-caught population was presumed to represent both species. The laboratory strain of D. simulans used was a $w$ stock that carried the endogenous wRi Wolbachia strain. The D. melanogaster strain used for the initial nutrient feeds and for crossing wMel Wolbachia into the other fly strains was $w$; $S p$ / Cyo; Sb/TM6B. Other D. melanogaster fly strains used were the gigas VALIUM20 TRiP line: $y$, $s c, v ; P\{$ TRiP.HMS01217\}attP2/TM3, Sb; the chico VALIUM20 TRiP line: $y, s c, v ; P\{T R i P$. HMS01553\}attP2/TM3, Sb; the somatic daughterless driver: $w$; $P\{w+, G M R 12 B 08-G A L 4\} a t t P 2$; the germline triple driver: $P\{$ otu-GAL4::VP16.1\}; P\{GAL4-Nos.NGT\}40; $P\{G A L 4:: V P 16-N o s$. UTR $\} M V D 1$; and the stocks used for IPC ablation: $w$; $P\{w+$, dilp2::GS-GAL4\}/Cyo, and $w$; $P\{w+, U A S:: R e a p e r\}$. During this work, $w \mathrm{Mel}$ was introduced into the somatic daughterless driver, the germline triple driver, and the dilp2::GS-GAL4 driver, and the infected versions of these stocks were crossed to the TRiP or UAS:Reaper responders. DrosDel isogenic flies carrying wMel were used for real-time quantitative PCR analyses [122].

\section{Food preparation and administration}

The standard food recipe used was based upon that of the Bloomington Drosophila Stock Center [127]. The food was prepared in large batches that consisted of 20L water, 337g yeast, $190 \mathrm{~g}$ soy flour, 1325g yellow corn meal, 96g agar, 1.5L Karo light corn syrup and $94 \mathrm{~mL}$ propionic acid. To create yeast paste for this study, live bakers yeast was mixed together with water to create a smooth, thick paste. To create the "control food" used in this study, we mixed together $1.5 \mathrm{~mL} \mathrm{ddH} 2 \mathrm{O}$ and $3.5 \mathrm{~mL}$ of melted standard food in a narrow-mouthed vial, then let cool in an ice bucket to solidify the food suspension. The same procedure applied to creation of all other nutrient-altered foods used in this study. For "corn-syrup-enriched" food condition, $1.5 \mathrm{~mL}$ Karo light corn syrup was used. For "yeast-enriched" food condition, $1.5 \mathrm{~mL}$ of heatkilled yeast paste was used. The "BSA-enriched" food carried $0.4 \mathrm{~g} \mathrm{BSA}, 1.5 \mathrm{~mL}$ water, and $3.5 \mathrm{~mL}$ standard food. For the "sucrose-enriched", "glucose-enriched" and "fructose-enriched" foods, fresh sugar solutions were prepared at a final concentration of $1 \mathrm{~g} / \mathrm{mL}$, then $1.5 \mathrm{~mL}$ of this concentrate was combined with $3.5 \mathrm{~mL}$ standard food for each vial. The "glucose + fructose enriched" condition carried $0.75 \mathrm{~mL}$ of $1 \mathrm{~g} / \mathrm{mL}$ glucose, $0.75 \mathrm{~mL} 1 \mathrm{~g} / \mathrm{mL}$ fructose, and $3.5 \mathrm{~mL}$ standard food. Alternate methods were used to prepare food for the other treatments. For the branched chain amino acid condition, the control condition contained $400 \mu \mathrm{L}$ water and $50 \mu \mathrm{L}$ DMSO mixed with $4.5 \mathrm{~mL}$ standard food, whereas the experimental condition carried $200 \mu \mathrm{L}$ of $1 \mathrm{mg} / \mathrm{mL}$ Arginine, $200 \mathrm{uL}$ of $1 \mathrm{mg} / \mathrm{mL}$ Isoleucine and $50 \mu \mathrm{L}$ DMSO mixed with $4.5 \mathrm{~mL}$ standard food. For the TORC1 testing, $50 \mu \mathrm{L}$ of either control DMSO or 30mM rapamycin/DMSO stock was mixed into $5 \mathrm{~mL}$ standard food. For tests of IPC function, $50 \mu \mathrm{L}$ of either control DMSO or a $10 \mathrm{mM}$ mifepristone-DMSO stock was mixed into $5 \mathrm{~mL}$ standard food.

Laboratory Drosophila stocks were maintained on standard food at $23-24^{\circ} \mathrm{C}$. Identical population density was used in all vials, and control and experimental conditions run in parallel. Flies of the genotype $w$; $S p / C y o$; $S b / T M 6 B$ were used in all imaging experiments that assessed nutrition as the only variable. In the cases where crosses were needed to drive expression from 
TRIP line stocks or the dilp2:GAL4 stocks were used, we performed all crosses using identical population density and female age distribution in all vials, with control crosses always run in parallel. Virgin female flies were collected during the first 3 days of eclosion only, then subjected to nutrient conditions. The procedure was to collect a range of $0-24$ hour old adults, age these young flies for 2 days on standard food, and expose to treatment conditions for 3 more days. The mixture of D. melanogaster and D. simulans flies collected from nature likely varied in age. These flies were also exposed to standard food for 2 days, and transferred to experimental food for 3 days. In the case of IPC ablation, the collected flies were allowed to mature 2 days, then transferred to mifepristone-containing food or DMSO control food. The flies were maintained on this food for 14 days, transferring the population to a fresh vial every 3 days of the treatment period. After this was completed, the flies were exposed to nutrient-altered food for 3 days.

\section{Tissue staining, imaging, and analysis}

Samples were prepared from a minimum of 10-15 flies per condition in each replicate. Ovary dissection, fixation, and propidium iodide staining were done as previously described in order to label germline Wolbachia nucleoids [38]. Ovarian tissues for all samples in each replicate were mounted on slides in parallel to ensure maximal consistency in sample compression between slide and coverslip. All samples were then imaged on a Leica SP2 confocal microscope at $63 \mathrm{X}$ magnification with $1.5 \mathrm{X}$ zoom. Experimental samples verified to exhibit the same degree of compression as the control sample were pursued further, while any experimental samples deviating from that were discarded. Z-series images were acquired from each egg chamber of interest at $1.5 \mu \mathrm{m}$ intervals. Uniform intensity settings were applied to all egg chambers imaged within each replicate. A minimum of 7-10 oocytes were ultimately imaged from each condition, with all experimental oocytes matched for morphological consistency against control oocytes of the same replicate. Using this rigorous method, significant fold-differences in Wolbachia titer were consistently identified between control and experimental conditions, regardless of the baseline quantity of Wolbachia detected in each replicate.

To quantify Wolbachia titer in the confocal images, we used established methods to identify the deepest possible focal plane where Wolbachia are clearly visible in all samples tested for each replicate [32]. The images were processed in Photoshop to remove everything from the images except oocyte Wolbachia, which were then quantified using the Analyze Particles feature in Image J. This analysis ultimately quantifies the Wolbachia nucleoids carried per oocyte, or per nurse cell, within a single, representative focal plane of each egg chamber. Although the graphical data displayed in the figures present all experimental averages as normalized against the control averages, all statistical calculations were run by comparing each condition only against controls that were run in parallel. Significant differences were indicated by ANOVA. A minimum of 2-3 replicates were performed for most germline staining experiments described in this study. The only exception was the experiment in which Wolbachia titer responses were analyzed in both brain and ovary tissues. In that case, single replicates were done for each type of tissue stained, with all conditions run in parallel.

To analyze Wolbachia titer by real-time quantitative PCR, single flies were homogenized with a pestle in $250 \mu \mathrm{l}$ of Tris $\mathrm{HCl} 0.1 \mathrm{M}$, EDTA $0.1 \mathrm{M}$ and SDS $1 \%$ (pH 9) and incubated for 30 minutes at $70^{\circ} \mathrm{C}$. After $35 \mu \mathrm{l}$ of KAc were added the sample was incubated 30 minutes on ice, centrifuged for 15 minutes at $13.000 \mathrm{rpm}$ at $4^{\circ} \mathrm{C}$ and the supernatant stored. Samples were diluted 100x for qPCR. qPCr was performed as described previously [122], using the CFX384 Real-Time PCR Detection System and iQ SYBR Green Supermix (both BioRad). The relative amount of Wolbachia was calculated with the Pfaffl method [128], using the primers for the 
gene wsp to determine Wolbachia DNA levels and primers for host Rpl32 and Actin5C genes to normalize male and female samples, respectively [122]. Data from males were analyzed using a linear model on the log of the relative wsp levels (Im in R) [129]. Data from females were analyzed using a mixed linear model on the logs of relative wsp levels (lmer in R).

To analyze Wolbachia in the Drosophila central nervous system, brains were dissected and fixed as previously described [101]. Brains were incubated in anti-rabbit wsp antibody + PBST $(0.1 \%$ Triton X-100) for 4 hours at room temperature or at least 12 hours at 4 degrees. For secondary antibody staining, goat anti-rabbit Alexa Fluor 546 (Invitrogen) was used at room temperature or at least 12 hours at four degrees. Actin labeling was done with phalloidin conjugated to Alexa 488, diluted 1:100 in PBST, for one hour at room temperature. Brain tissues were imaged on a Leica SP2 confocal microscope at $63 \mathrm{X}$ magnification. Brains were quantified with Leica LAF AS software. One representative focal plane per brain was scored. Cells containing one or more Wolbachia were scored as infected. Wolbachia aggregates larger than 10 microns $^{2}$ were scored as a "cluster" [101].

To assess Wolbachia nucleoid shape, we acquired Z-series images of stage 10A oocytes at $63 \mathrm{X}$ magnification with $5 \mathrm{X}$ zoom. Then we created a projection of 4 images from each Z-series, located just beneath the follicle cell layer, and measured the length of individual nucleoids using the "line" tool located within the Profile function of Quantification Tools in the Leica SP2 software. Elongation index was calculated as a function of length divided by width. It is assumed that the bacteria are random in orientation, and thus detecting a range of nucleoid morphologies ranging from spherical to rod-shaped is possible. Chi square tests were used to compare Wolbachia length and elongation index exhibited by bacterial populations from each treatment condition.

\section{Supporting Information}

S1 Table. Nutritional content of the food types administered. This table displays combined information from the USDA National Nutrient Database for Standard Reference, Release 27, scaled to the volumes of ingredients used for each condition. The protein content of the branched chain amino acid (BCAA)-enriched food, noted with an asterisk, represents the combined weight of the added amino acids plus other protein present in the food. The nutritional content of glucose-enriched, fructose-enriched, and glucose+fructose enriched food were nearly identical to sucrose-enriched food according to the nutrient classifications used in this table, and thus are not shown.

S1 Fig. Host diet affects oocyte Wolbachia titer in wild-caught Drosophila. Wolbachia nucleoids were quantified in the oocytes of wild-caught D. melanogaster and D. simulans. Control and yeast-enriched feeding conditions were used. ${ }^{*}$ indicates a significant change in titer. (TIF)

S2 Fig. BSA-enriched food has no impact on oocyte Wolbachia titer. Female D. melanogaster were exposed in parallel to control and BSA-enriched food conditions, and their Wolbachia nucleoids were quantified in oogenesis. Average titer levels are shown. (TIF)

S3 Fig. Oocyte Wolbachia titer is unaffected by mating. Oocyte Wolbachia nucleoids were quantified in D. melanogaster females that had either been reared together with males or maintained in isolation from males. Average titer levels are shown.

(TIF) 
S4 Fig. Dietary conditions affect Wolbachia nucleoid morphology. A-C) Zoomed-in views of Wolbachia nucleoids in D. melanogaster oocytes. Treatments: A) Control fly food. B) Yeastenriched food. C) Sucrose-enriched food. D) Assessment of Wolbachia nucleoid length in response to nutrient conditions. E) Quantification of elongation index exhibited by the same bacteria. ${ }^{*}$ indicates a significant change in titer. Scale bar: $10 \mu \mathrm{m}$.

(TIF)

S5 Fig. Nutrient-altered food affects oocyte Wolbachia titer in D. simulans. The D. simulans flies used for this preparation were raised, exposed to nutrient-altered food, and stained in parallel with the D. simulans analyzed in Fig. 6 A-F'. ${ }^{*}$ indicates a significant change in titer. (TIF)

\section{Acknowledgments}

We thank the Sullivan lab, Bill Saxton, Jian Cao, Henri Jasper, Fernando Noriega, Marcela Nouzova, Aaron Neiman, Babak Ebrahimi, Inna Djagaeva, Adan Codina, Steen Christensen, Alejandro Barbieri, Christopher Chin, Matthew DeGennaro, Angeline Lim, Bill Ja, Catharina Lindley, Malika Bell, Yulianna Ortega, Gerhardt Haupt and the Wolbachia community for the shared discussions, fly strains, reagents and technical help. We especially thank the Bloomington Drosophila Stock Center and the TRiP project at Harvard Medical School for providing transgenic RNAi fly stocks used in this study.

\section{Author Contributions}

Conceived and designed the experiments: LRS PMW JPS AR LT RA WS. Performed the experiments: LRS PMW JPS AR LT RA. Analyzed the data: LRS PMW JPS AR LT RA. Contributed reagents/materials/analysis tools: LRS LT WS. Wrote the paper: LRS PMW JPS LT RA WS.

\section{References}

1. Davy SK, Allemand D, Weis VM (2012) Cell biology of cnidarian-dinoflagellate symbiosis. Microbiol Mol Biol Rev 76: 229-261. doi: 10.1128/MMBR.05014-11 PMID: 22688813

2. Feldhaar H, Straka J, Krischke M, Berthold K, Stoll S, et al. (2007) Nutritional upgrading for omnivorous carpenter ants by the endosymbiont Blochmannia. BMC Biol 5: 48. PMID: 17971224

3. Gibson KE, Kobayashi H, Walker GC (2008) Molecular Determinants of a Symbiotic Chronic Infection. Annual Review of Genetics 42: 413-441. doi: 10.1146/annurev.genet.42.110807.091427 PMID: 18983260

4. Hosokawa T, Koga R, Kikuchi Y, Meng XY, Fukatsu T (2010) Wolbachia as a bacteriocyte-associated nutritional mutualist. Proc Natl Acad Sci U S A 107: 769-774. doi: 10.1073/pnas.0911476107 PMID: 20080750

5. Johnson MD (2011) The acquisition of phototrophy: adaptive strategies of hosting endosymbionts and organelles. Photosynthesis Research 107: 117-132. doi: 10.1007/s11120-010-9546-8 PMID: 20405214

6. Nakabachi A, Ishikawa $\mathrm{H}$ (1999) Provision of riboflavin to the host aphid, Acyrthosiphon pisum, by endosymbiotic bacteria, Buchnera. J Insect Physiol 45: 1-6. PMID: 12770389

7. Nogge G (1981) Significance of Symbionts for the Maintenance of an Optimal Nutritional State for Successful Reproduction in Hematophagous Arthropods. Parasitology 82: 101-104.

8. Oldroyd GED (2013) Speak, friend, and enter: signalling systems that promote beneficial symbiotic associations in plants. Nature Reviews Microbiology 11: 252-263. doi: 10.1038/nrmicro2990 PMID 23493145

9. Parniske $M(2008)$ Arbuscular mycorrhiza: the mother of plant root endosymbioses. Nature Reviews Microbiology 6: 763-775. doi: 10.1038/nrmicro1987 PMID: 18794914

10. Puchta O (1955) Experimentelle Untersuchungen uber die Bedeutung der Symbiose der Kleiderlaus Pediculus vestimenti Burm. Z Parasitenk 17. PMID: 13312506 
11. Sabree ZL, Huang CY, Okusu A, Moran NA, Normark BB (2013) The nutrient supplying capabilities of Uzinura, an endosymbiont of armoured scale insects. Environ Microbiol 15: 1988-1999. doi: 10. 1111/1462-2920.12058 PMID: 23279075

12. Sabree ZL, Kambhampati S, Moran NA (2009) Nitrogen recycling and nutritional provisioning by Blattabacterium, the cockroach endosymbiont. Proc Natl Acad Sci U S A 106: 19521-19526. doi: 10. 1073/pnas.0907504106 PMID: 19880743

13. Shigenobu S, Watanabe H, Hattori M, Sakaki Y, Ishikawa $H$ (2000) Genome sequence of the endocellular bacterial symbiont of aphids Buchnera sp. APS. Nature 407: 81-86. PMID: 10993077

14. Stambler N (2011) Zooxanthellae: The yellow symbionts inside animals. In: Dubinsy Z, Stambler N, editors. Coral reefs: an ecosystem in transition. New York: Springer. pp. 87-106.

15. Snyder AK, McLain C, Rio RVM (2012) The Tsetse Fly Obligate Mutualist Wigglesworthia morsitans Alters Gene Expression and Population Density via Exogenous Nutrient Provisioning. Applied and Environmental Microbiology 78: 7792-7797. doi: 10.1128/AEM.02052-12 PMID: 22904061

16. Snyder AK, Deberry JW, Runyen-Janecky L, Rio RV (2010) Nutrient provisioning facilitates homeostasis between tsetse fly (Diptera: Glossinidae) symbionts. Proc Biol Sci 277: 2389-2397. doi: 10. 1098/rspb.2010.0364 PMID: 20356887

17. Werren JH, Baldo L, Clark ME (2008) Wolbachia: master manipulators of invertebrate biology. Nat Rev Microbiol 6: 741-751. doi: 10.1038/nrmicro1969 PMID: 18794912

18. Serbus LR, Casper-Lindley C, Landmann F, Sullivan W (2008) The Genetics and Cell Biology of Wolbachia-Host Interactions. Annual Review of Genetics 42: 683-707. doi: 10.1146/annurev.genet.41. 110306.130354 PMID: 18713031

19. Zug R, Hammerstein P (2012) Still a host of hosts for Wolbachia: analysis of recent data suggests that $40 \%$ of terrestrial arthropod species are infected. PLoS One 7: e38544. doi: 10.1371/journal.pone. 0038544 PMID: 22685581

20. Ashburner M (1989) Drosophila, a Laboratory Handbook. New York: Cold Spring Harbor Laboratory Press. $1331 \mathrm{p}$.

21. Snook RR, Cleland SY, Wolfner MF, Karr TL (2000) Offsetting effects of Wolbachia infection and heat shock on sperm production in Drosophila simulans: analyses of fecundity, fertility and accessory gland proteins. Genetics 155: 167-178. PMID: 10790392

22. Bressac C, Rousset $F(1993)$ The reproductive incompatibility system in Drosophila simulans: DAPIstaining analysis of the Wolbachia symbionts in sperm cysts. J Invertebr Pathol 61: 226-230. PMID: 7689622

23. Clark ME, Veneti Z, Bourtzis K, Karr TL (2002) The distribution and proliferation of the intracellular bacteria Wolbachia during spermatogenesis in Drosophila. Mech Dev 111: 3-15. PMID: 11804774

24. Hoffmann AA, Hercus M, Dagher H (1998) Population dynamics of the Wolbachia infection causing cytoplasmic incompatibility in Drosophila melanogaster. Genetics 148: 221-231. PMID: 9475734

25. Turelli M, Hoffmann AA (1995) Cytoplasmic incompatibility in Drosophila simulans: dynamics and parameter estimates from natural populations. Genetics 140: 1319-1338. PMID: 7498773

26. Ashburner M (1989) Developmental Biology. Drosophila, a Laboratory Handbook. Cold Spring Harbor: Cold Spring Harbor Laboratory Press. pp. 139-204.

27. King RC (1970) Ovarian development in Drosophila melanogaster. New York: Academic Press. 227 p.

28. Spradling AC (1993) Developmental Genetics of Oogenesis. In: Bate M, Arias AM, editors. The Development of Drosophila melanogaster. New York: Cold Spring Harbor Laboratory Press. pp. 1-70.

29. Kugler JM, Lasko $P$ (2009) Localization, anchoring and translational control of oskar, gurken, bicoid and nanos mRNA during Drosophila oogenesis. Fly (Austin) 3: 15-28. PMID: 19182536

30. Hudson AM, Cooley L (2014) Methods for studying oogenesis. Methods.

31. Ferree PM, Frydman HM, Li JM, Cao J, Wieschaus E, et al. (2005) Wolbachia utilizes host microtubules and Dynein for anterior localization in the Drosophila oocyte. PLoS Pathog 1: e14. PMID: 16228015

32. Serbus L, Ferreccio A, Zhukova M, McMorris C, Kiseleva E, et al. (2011) A feedback loop between Wolbachia and the Drosophila gurken mRNP complex influences Wolbachia titer. J Cell Sci 124: 4299-4308. doi: 10.1242/jcs.092510 PMID: 22193955

33. Casper-Lindley C, Kimura S, Saxton DS, Essaw Y, Simpson I, et al. (2011) Rapid FluorescenceBased Screening for Wolbachia Endosymbionts in Drosophila Germ Line and Somatic Tissues. Applied and Environmental Microbiology 77: 4788-4794. doi: 10.1128/AEM.00215-11 PMID: 21622788 
34. Fast EM, Toomey ME, Panaram K, Desjardins D, Kolaczyk ED, et al. (2011) Wolbachia Enhance Drosophila Stem Cell Proliferation and Target the Germline Stem Cell Niche. Science 334: 990-992. doi: 10.1126/science.1209609 PMID: 22021671

35. Toomey ME, Panaram K, Fast EM, Beatty C, Frydman HM (2013) Evolutionarily conserved Wolbachia-encoded factors control pattern of stem-cell niche tropism in Drosophila ovaries and favor infection. Proceedings of the National Academy of Sciences of the United States of America 110: 1078810793. doi: 10.1073/pnas.1301524110 PMID: 23744038

36. Frydman HM, Li JM, Robson DN, Wieschaus E (2006) Somatic stem cell niche tropism in Wolbachia. Nature 441: 509-512. PMID: 16724067

37. Veneti Z, Clark ME, Karr TL, Savakis C, Bourtzis K (2004) Heads or tails: host-parasite interactions in the Drosophila-Wolbachia system. Appl Environ Microbiol 70: 5366-5372. PMID: 15345422

38. Serbus LR, Sullivan W (2007) A Cellular Basis for Wolbachia Recruitment to the Host Germline. PLoS Pathog 3: e190. PMID: 18085821

39. Hadfield SJ, Axton JM (1999) Germ cells colonized by endosymbiotic bacteria. Nature 402: 482 . PMID: 10591206

40. Anagnostou C, Dorsch M, Rohlfs M (2010) Influence of dietary yeasts on Drosophila melanogaster life-history traits. Entomol Exp Appl 136: 1-11.

41. Begon M (1982) Yeasts and Drosophila. In: Ashburner M, Carson HL, Thompson JN Jr., editors. The Genetics and Biology of Drosophila. San Francisco: Academic Press. pp. 345-384.

42. Shorrocks B (1982) The Breeding Sites of Temperate Woodland Drosophila. In: Ashburner M, Carson HL, Thompson JN Jr., editors. The Genetics and Biology and Biology of Drosophila. San Francisco: Academic Press. pp. 385-428.

43. Brncic D (1983) Ecology of Flower-Breeding Drosophila. In: Ashburner M, Carson HL, Thompson JN Jr., editors. The Genetics and Biology of Drosophila. San Francisco: Academic Press. pp. 333-382.

44. Kukor JJ, Martin MM (1987) Nutritional Ecology of Fungus-feeding Arthropods. In: Slansky F Jr., Rodriguez JG, editors. Nutritional Ecology of Insects, Mites, Spiders, and Related Invertebrates. New York: John Wiley and Sons. pp. 791-836.

45. Coluccio AE, Rodriguez RK, Kernan MJ, Neiman AM (2008) The yeast spore wall enables spores to survive passage through the digestive tract of Drosophila. PLoS One 3: e2873. doi: 10.1371/journal pone.0002873 PMID: 18682732

46. Teleman AA (2010) Molecular mechanisms of metabolic regulation by insulin in Drosophila. Biochemical Journal 425: 13-26. doi: 10.1042/BJ20091181 PMID: 20001959

47. Colombani J, Raisin S, Pantalacci S, Radimerski T, Montagne J, et al. (2003) A nutrient sensor mechanism controls Drosophila growth. Cell 114: 739-749. PMID: 14505573

48. Colombani J, Raisin S, Pantalacci S, Radimerski T, Montagne J, et al. (2003) Amino acids and the humoral regulation of growth: fat bodies use Slimfast (vol 114, pg 656, 2003). Cell 115: 123-123.

49. Choi JW, Chen J, Schreiber SL, Clardy J (1996) Structure of the FKBP12-rapamycin complex interacting with the binding domain of human FRAP. Science 273: 239-242. PMID: 8662507

50. Chen J, Zheng XF, Brown EJ, Schreiber SL (1995) Identification of an 11-Kda Fkbp12-RapamycinBinding Domain within the 289-Kda Fkbp12-Rapamycin-Associated Protein and Characterization of a Critical Serine Residue. Proceedings of the National Academy of Sciences of the United States of America 92: 4947-4951. PMID: 7539137

51. Guertin DA, Sabatini DM (2009) The Pharmacology of mTOR Inhibition. Science Signaling 2.

52. Yip CK, Murata K, Walz T, Sabatini DM, Kang SA (2010) Structure of the Human mTOR Complex I and Its Implications for Rapamycin Inhibition. Molecular Cell 38: 768-774. doi: 10.1016/j.molcel. 2010.05.017 PMID: 20542007

53. Gonzalez IM, Martin PM, Burdsal C, Sloan JL, Mager S, et al. (2012) Leucine and arginine regulate trophoblast motility through mTOR-dependent and independent pathways in the preimplantation mouse embryo. Developmental Biology 361: 286-300. doi: 10.1016/j.ydbio.2011.10.021 PMID: 22056783

54. Wang YX, Zhang LL, Zhou GL, Liao ZY, Ahmad H, et al. (2012) Dietary L-arginine supplementation improves the intestinal development through increasing mucosal Akt and mammalian target of rapamycin signals in intra-uterine growth retarded piglets. British Journal of Nutrition 108: 1371-1381. doi: 10.1017/S0007114511006763 PMID: 22217383

55. Xi PB, Jiang ZY, Dai ZL, Li XL, Yao K, et al. (2010) Regulation of protein turnover in porcine intestinal cells by L-glutamine (GIn). Faseb Journal 24. 
56. Yao K, Yin YL, Chu WY, Li ZQ, Deng D, et al. (2008) Dietary arginine supplementation increases mTOR signaling activity in skeletal muscle of neonatal pigs. Journal of Nutrition 138: 867-872. PMID: 18424593

57. Atherton PJ, Smith K, Etheridge T, Rankin D, Rennie MJ (2010) Distinct anabolic signalling responses to amino acids in C2C12 skeletal muscle cells. Amino Acids 38: 1533-1539. doi: 10.1007/s00726009-0377-x PMID: 19882215

58. Norton LE, Layman DK, Bunpo P, Anthony TG, Brana DV, et al. (2009) The Leucine Content of a Complete Meal Directs Peak Activation but Not Duration of Skeletal Muscle Protein Synthesis and Mammalian Target of Rapamycin Signaling in Rats. Journal of Nutrition 139: 1103-1109. doi: 10. 3945/jn.108.103853 PMID: 19403715

59. Dibble CC, Elis W, Menon S, Qin W, Klekota J, et al. (2012) TBC1D7 Is a Third Subunit of the TSC1TSC2 Complex Upstream of mTORC1. Molecular Cell 47: 535-546. doi: 10.1016/j.molcel.2012.06. 009 PMID: 22795129

60. Stocker H, Radimerski T, Schindelholz B, Wittwer F, Belawat P, et al. (2003) Rheb is an essential regulator of S6K in controlling cell growth in Drosophila. Nature Cell Biology 5: 559-565. PMID: 12766775

61. Saucedo LJ, Gao XS, Chiarelli DA, Li L, Pan D, et al. (2003) Rheb promotes cell growth as a component of the insulin/TOR signalling network. Nature Cell Biology 5: 566-571. PMID: 12766776

62. Inoki K, Li Y, Zhu TQ, Wu J, Guan KL (2002) TSC2 is phosphorylated and inhibited by Akt and suppresses mTOR signalling. Nature Cell Biology 4: 648-657. PMID: 12172553

63. Cai SL, Tee AR, Short JD, Bergeron JM, Kim J, et al. (2006) Activity of TSC2 is inhibited by AKT-mediated phosphorylation and membrane partitioning. Journal of Cell Biology 173: 279-289. PMID: 16636147

64. Ito N, Rubin GM (1999) Gigas, a Drosophila homolog of tuberous sclerosis gene product-2, regulates the cell cycle. Cell 96: 529-539. PMID: 10052455

65. Huang JX, Manning BD (2009) A complex interplay between Akt, TSC2 and the two mTOR complexes. Biochemical Society Transactions 37: 217-222. doi: 10.1042/BST0370217 PMID: 19143635

66. Inoki K, Guan KL (2009) Tuberous sclerosis complex, implication from a rare genetic disease to common cancer treatment. Human Molecular Genetics 18: R94-R100. doi: 10.1093/hmg/ddp032 PMID: 19297407

67. Tapon N, Ito N, Dickson BJ, Treisman JE, Hariharan IK (2001) The Drosophila tuberous sclerosis complex gene homologs restrict cell growth and cell proliferation. Cell 105: 345-355. PMID: 11348591

68. Gao XS, Pan DJ (2001) TSC1 and TSC2 tumor suppressors antagonize insulin signaling in cell growth. Genes \& Development 15: 1383-1392.

69. Ni JQ, Zhou R, Czech B, Liu LP, Holderbaum L, et al. (2011) A genome-scale shRNA resource for transgenic RNAi in Drosophila. Nat Methods 8: 405-407. doi: 10.1038/nmeth.1592 PMID: 21460824

70. Ni JQ, Markstein M, Binari R, Pfeiffer B, Liu LP, et al. (2008) Vector and parameters for targeted transgenic RNA interference in Drosophila melanogaster. Nat Methods 5: 49-51. PMID: 18084299

71. Wodarz A, Hinz U, Engelbert M, Knust E (1995) Expression of crumbs confers apical character on plasma membrane domains of ectodermal epithelia of Drosophila. Cell 82: 67-76. PMID: 7606787

72. Petrella LN, Smith-Leiker T, Cooley $L$ (2007) The Ovhts polyprotein is cleaved to produce fusome and ring canal proteins required for Drosophila oogenesis. Development 134: 702-712.

73. Broughton SJ, Piper MDW, Ikeya T, Bass TM, Jacobson J, et al. (2005) Longer lifespan, altered metabolism, and stress resistance in Drosophila from ablation of cells making insulin-like ligands. Proceedings of the National Academy of Sciences of the United States of America 102: 3105-3110. PMID: 15708981

74. Haselton A, Sharmin E, Schrader J, Sah M, Poon P, et al. (2010) Partial ablation of adult Drosophila insulin-producing neurons modulates glucose homeostasis and extends life span without insulin resistance. Cell Cycle 9: 3063-3071. doi: 10.4161/cc.9.15.12458 PMID: 20699643

75. Pasco MY, Leopold $P(2012)$ High sugar-induced insulin resistance in Drosophila relies on the lipocalin Neural Lazarillo. PLoS One 7: e36583. doi: 10.1371/journal.pone.0036583 PMID: 22567167

76. Morris SN, Coogan C, Chamseddin K, Fernandez-Kim SO, Kolli S, et al. (2012) Development of dietinduced insulin resistance in adult Drosophila melanogaster. Biochim Biophys Acta 1822: 12301237. doi: 10.1016/j.bbadis.2012.04.012 PMID: 22542511

77. Yang Q, Graham TE, Mody N, Preitner F, Peroni OD, et al. (2005) Serum retinol binding protein 4 contributes to insulin resistance in obesity and type 2 diabetes. Nature 436: 356-362. PMID: 16034410 
78. Graham TE, Yang Q, Bluher M, Hammarstedt A, Ciaraldi TP, et al. (2006) Retinol-binding protein 4 and insulin resistance in lean, obese, and diabetic subjects. N Engl J Med 354:2552-2563. PMID: 16775236

79. Norseen J, Hosooka T, Hammarstedt A, Yore MM, Kant S, et al. (2012) Retinol-binding protein 4 inhibits insulin signaling in adipocytes by inducing proinflammatory cytokines in macrophages through a cJun N-terminal kinase- and toll-like receptor 4-dependent and retinol-independent mechanism. Mol Cell Biol 32: 2010-2019. doi: 10.1128/MCB.06193-11 PMID: 22431523

80. Bohni R, Riesgo-Escovar J, Oldham S, Brogiolo W, Stocker H, et al. (1999) Autonomous control of cell and organ size by CHICO, a Drosophila homolog of vertebrate IRS1-4. Cell 97: 865-875. PMID: 10399915

81. Ogawa W, Matozaki T, Kasuga M (1998) Role of binding proteins to IRS-1 in insulin signalling. Molecular and Cellular Biochemistry 182: 13-22. PMID: 9609110

82. Werz C, Kohler K, Hafen E, Stocker H (2009) The Drosophila SH2B family adaptor Lnk acts in parallel to chico in the insulin signaling pathway. PLoS Genet 5: e1000596. doi: 10.1371/journal.pgen. 1000596 PMID: 19680438

83. Drummond-Barbosa D, Spradling AC (2001) Stem cells and their progeny respond to nutritional changes during Drosophila oogenesis. Dev Biol 231: 265-278. PMID: 11180967

84. Tatar M, Kopelman A, Epstein D, Tu MP, Yin CM, et al. (2001) A mutant Drosophila insulin receptor homolog that extends life-span and impairs neuroendocrine function. Science 292: 107-110. PMID: 11292875

85. Richard DS, Rybczynski R, Wilson TG, Wang Y, Wayne ML, et al. (2005) Insulin signaling is necessary for vitellogenesis in Drosophila melanogaster independent of the roles of juvenile hormone and ecdysteroids: female sterility of the chico1 insulin signaling mutation is autonomous to the ovary. $\mathrm{J}$ Insect Physiol 51: 455-464. PMID: 15890189

86. LaFever L, Drummond-Barbosa D (2005) Direct control of germline stem cell division and cyst growth by neural insulin in Drosophila. Science 309: 1071-1073. PMID: 16099985

87. Ikeya T, Broughton S, Alic N, Grandison R, Partridge L (2009) The endosymbiont Wolbachia increases insulin/IGF-like signalling in Drosophila. Proceedings of the Royal Society B-Biological Sciences 276: 3799-3807. doi: 10.1098/rspb.2009.0778 PMID: 19692410

88. LaFever L, Feoktistov A, Hsu HJ, Drummond-Barbosa D (2010) Specific roles of Target of rapamycin in the control of stem cells and their progeny in the Drosophila ovary (vol 137, pg 2117, 2010). Development 137: 2451-2451.

89. Gronke S, Clarke DF, Broughton S, Andrews TD, Partridge L (2010) Molecular Evolution and Functional Characterization of Drosophila Insulin-Like Peptides. Plos Genetics 6.

90. Hsu HJ, Drummond-Barbosa D (2009) Insulin levels control female germline stem cell maintenance via the niche in Drosophila. Proceedings of the National Academy of Sciences of the United States of America 106: 1117-1121. doi: 10.1073/pnas.0809144106 PMID: 19136634

91. Hsu HJ, LaFever L, Drummond-Barbosa D (2008) Diet controls normal and tumorous germline stem cells via insulin-dependent and-independent mechanisms in Drosophila. Developmental Biology 313: 700-712. PMID: 18068153

92. Heifetz Y, Tram U, Wolfner MF (2001) Male contributions to egg production: the role of accessory gland products and sperm in Drosophila melanogaster. Proc Biol Sci 268: 175-180. PMID: 11209888

93. Soller M, Bownes M, Kubli E (1997) Mating and sex peptide stimulate the accumulation of yolk in oocytes of Drosophila melanogaster. Eur J Biochem 243: 732-738. PMID: 9057839

94. Soller M, Bownes M, Kubli E (1999) Control of oocyte maturation in sexually mature Drosophila females. Dev Biol 208: 337-351. PMID: 10191049

95. King RC, Sang JH (1959) Oogenesis in adult Drosophila melanogaster. VIII. The role of folic acid in oogenesis. Growth 23: 37-53. PMID: 13672469

96. Carvalho GB, Kapahi P, Anderson DJ, Benzer S (2006) Allocrine modulation of feeding behavior by the Sex Peptide of Drosophila. Curr Biol 16: 692-696. PMID: 16581515

97. Landmann F, Bain O, Martin C, Uni S, Taylor M, et al. (2012) Both asymmetric mitotic segregation and cell-to-cell invasion are required for stable germline transmission of Wolbachia in filarial nematodes. Biology Open 00: 1-12.

98. Pierce A, Gillette D, Jones PG (2011) Escherichia coli cold shock protein CsdA effects an increase in septation and the resultant formation of coccobacilli at low temperature. Arch Microbiol 193: 373384. doi: 10.1007/s00203-011-0682-0 PMID: 21359956

99. Frenkiel-Krispin D, Minsky A (2006) Nucleoid organization and the maintenance of DNA integrity in E. coli, B. subtilis and D. radiodurans. J Struct Biol 156: 311-319. PMID: 16935006 
100. Min KT, Benzer S (1997) Wolbachia, normally a symbiont of Drosophila, can be virulent, causing degeneration and early death. Proceedings of the National Academy of Sciences of the United States of America 94: 10792-10796. PMID: 9380712

101. Albertson R, Tan V, Leads RR, Reyes M, Sullivan W, et al. (2013) Mapping Wolbachia distributions in the adult Drosophila brain. Cellular Microbiology 15: 1527-1544. doi: 10.1111/cmi.12136 PMID: 23490256

102. Wu M, Sun LV, Vamathevan J, Riegler M, Deboy R, et al. (2004) Phylogenomics of the reproductive parasite Wolbachia pipientis wMel: a streamlined genome overrun by mobile genetic elements. PLoS Biol 2: E69. PMID: 15024419

103. Caragata EP, Rances E, O'Neill SL, McGraw EA (2014) Competition for amino acids between Wolbachia and the mosquito host, Aedes aegypti. Microb Ecol 67: 205-218. doi: 10.1007/s00248-0130339-4 PMID: 24337107

104. Markov AV, Zakharov IA (2006) The parasitic bacterium Wolbachia and the origin of the eukaryotic cell. Paleontological Journal 40: 115-124.

105. Ponton F, Wilson K, Holmes A, Raubenheimer D, Robinson KL, et al. (2015) Macronutrients mediate the functional relationship between Drosophila and Wolbachia. Proc Biol Sci 282.

106. Ikeya T, Broughton S, Alic N, Grandison R, Partridge L (2009) The endosymbiont Wolbachia increases insulin/IGF-like signalling in Drosophila. Proc Biol Sci 276: 3799-3807. doi: 10.1098/rspb. 2009.0778 PMID: 19692410

107. Matilainen $\mathrm{O}$, Arpalahti L, Rantanen V, Hautaniemi S, Holmberg Cl (2013) Insulin/IGF-1 signaling regulates proteasome activity through the deubiquitinating enzyme UBH-4. Cell Rep 3: 1980-1995. doi: 10.1016/j.celrep.2013.05.012 PMID: 23770237

108. Blakesley VA, Koval AP, Stannard BS, Scrimgeour A, LeRoith D (1998) Replacement of tyrosine 1251 in the carboxyl terminus of the insulin-like growth factor-I receptor disrupts the actin cytoskeleton and inhibits proliferation and anchorage-independent growth. J Biol Chem 273: 18411-18422. PMID: 9660809

109. Coletta DK, Mandarino LJ (2011) Mitochondrial dysfunction and insulin resistance from the outside in: extracellular matrix, the cytoskeleton, and mitochondria. Am J Physiol Endocrinol Metab 301: E749755. doi: 10.1152/ajpendo.00363.2011 PMID: 21862724

110. Hwang H, Bowen BP, Lefort N, Flynn CR, De Filippis EA, et al. (2010) Proteomics analysis of human skeletal muscle reveals novel abnormalities in obesity and type 2 diabetes. Diabetes 59: 33-42. doi: 10.2337/db09-0214 PMID: 19833877

111. Abe Y, Yoon SO, Kubota K, Mendoza MC, Gygi SP, et al. (2009) p90 ribosomal S6 kinase and p70 ribosomal S6 kinase link phosphorylation of the eukaryotic chaperonin containing TCP-1 to growth factor, insulin, and nutrient signaling. J Biol Chem 284: 14939-14948. doi: 10.1074/jbc.M900097200 PMID: 19332537

112. Huang J, Brumell JH (2014) Bacteria-autophagy interplay: a battle for survival. Nat Rev Microbiol 12 : 101-114. doi: 10.1038/nrmicro3160 PMID: 24384599

113. Steele S, Brunton J, Ziehr B, Taft-Benz S, Moorman N, et al. (2013) Francisella tularensis harvests nutrients derived via ATG5-independent autophagy to support intracellular growth. PLoS Pathog 9: e1003562. doi: 10.1371/journal.ppat.1003562 PMID: 23966861

114. Yu HB, Croxen MA, Marchiando AM, Ferreira RB, Cadwell K, et al. (2014) Autophagy facilitates Salmonella replication in HeLa cells. MBio 5: e00865-00814. doi: 10.1128/mBio.00865-14 PMID: 24618251

115. Voronin D, Cook DAN, Steven A, Taylor MJ (2012) Autophagy regulates Wolbachia populations across diverse symbiotic associations. Proceedings of the National Academy of Sciences of the United States of America 109: E1638-E1646. doi: 10.1073/pnas.1203519109 PMID: 22645363

116. Hedges LM, Brownlie JC, O'Neill SL, Johnson KN (2008) Wolbachia and virus protection in insects. Science 322: 702. doi: 10.1126/science.1162418 PMID: 18974344

117. Teixeira L, Ferreira A, Ashburner M (2008) The bacterial symbiont Wolbachia induces resistance to RNA viral infections in Drosophila melanogaster. PLoS Biol 6: e2. doi: 10.1371/journal.pbio.1000002 PMID: 19222304

118. Rainey SM, Shah P, Kohl A, Dietrich I (2014) Understanding the Wolbachia-mediated inhibition of arboviruses in mosquitoes: progress and challenges. J Gen Virol 95: 517-530. doi: 10.1099/vir.0. 057422-0 PMID: 24343914

119. Kriesner P, Hoffmann AA, Lee SF, Turelli M, Weeks AR (2013) Rapid sequential spread of two Wolbachia variants in Drosophila simulans. PLoS Pathog 9: e1003607. doi: 10.1371/journal.ppat.1003607 PMID: 24068927 
120. Hoffmann AA, Montgomery BL, Popovici J, Iturbe-Ormaetxe I, Johnson PH, et al. (2011) Successful establishment of Wolbachia in Aedes populations to suppress dengue transmission. Nature 476: 454-457. doi: 10.1038/nature10356 PMID: 21866160

121. Walker T, Johnson PH, Moreira LA, Iturbe-Ormaetxe I, Frentiu FD, et al. (2011) The wMel Wolbachia strain blocks dengue and invades caged Aedes aegypti populations. Nature 476: 450-453. doi: 10. 1038/nature10355 PMID: 21866159

122. Chrostek E, Marialva MSP, Esteves SS, Weinert LA, Martinez J, et al. (2013) Wolbachia Variants Induce Differential Protection to Viruses in Drosophila melanogaster: A Phenotypic and Phylogenomic Analysis. Plos Genetics 9.

123. Lu P, Bian G, Pan X, Xi Z (2012) Wolbachia induces density-dependent inhibition to dengue virus in mosquito cells. PLoS Negl Trop Dis 6: e1754. doi: 10.1371/journal.pntd.0001754 PMID: 22848774

124. Osborne SE, Iturbe-Ormaetxe I, Brownlie JC, O'Neill SL, Johnson KN (2012) Antiviral protection and the importance of Wolbachia density and tissue tropism in Drosophila simulans. Appl Environ Microbiol 78: 6922-6929. doi: 10.1128/AEM.01727-12 PMID: 22843518

125. Osborne SE, Leong YS, O'Neill SL, Johnson KN (2009) Variation in antiviral protection mediated by different Wolbachia strains in Drosophila simulans. PLoS Pathog 5: e1000656. doi: 10.1371/journal. ppat.1000656 PMID: 19911047

126. Chrostek E, Marialva MS, Yamada R, O'Neill SL, Teixeira L (2014) High anti-viral protection without immune upregulation after interspecies Wolbachia transfer. PLoS One 9: e99025. doi: 10.1371/ journal.pone.0099025 PMID: 24911519

127. Bloomington_Drosophila_Stock_Center http://flystocks.bio.indiana.edu/Fly_Work/media-recipes/ bloomfood.htm.

128. Pfaffl MW (2001) A new mathematical model for relative quantification in real-time RT-PCR. Nucleic Acids Res 29: e45. PMID: 11328886

129. R_Development_Core_Team (2011) A language and Environment for Statistical Computing. Vienna, Austria: The R Foundation for Statistical Computing. doi: 10.1016/j.neuroimage.2011.01.013 PMID: 21238596 